\title{
Vector dark matter production from catalyzed annihilation
}

\author{
Chengfeng Cai and Hong-Hao Zhang ${ }^{1}$ \\ School of Physics, Sun Yat-Sen University, \\ Guangzhou 510275, China \\ E-mail: caichf3@mail.sysu.edu.cn, zhh98@mail.sysu.edu.cn
}

ABSTRACT: We provide a simple model of vector dark matter (DM) which can realize the recently proposed freeze-out mechanism with catalyzed annihilation. In our setup, a vector DM field $X_{\mu}$ and a catalyst field $C_{\mu}$ is unified by an $\mathrm{SU}(2)_{D}$ gauge symmetry. These gauge fields acquire their masses via spontaneously symmetry breaking triggered by a doublet and a real triplet scalar fields. The catalyst particle is automatically lighter than the DM since it only acquires mass from the vacuum expectation value of the doublet scalar. We also introduce a dimension-5 operator to generate a kinetic mixing term between $C_{\mu}$ and the $\mathrm{U}(1)_{Y}$ gauge field $B_{\mu}$. This mixing term is naturally small due to a suppression with a high UV completion scale, and thus it allows the catalyst to decay after the DM freeze-out. We derive the annihilation cross sections of processes $X^{*}+X \rightarrow 2 C$ and $3 C \rightarrow X^{*}+X$ and solve the Boltzmann equations for both the DM and the catalyst. We develop the analytical approximate solutions of the equations and find them matching the numerical solutions well. Constraints from relic abundance and indirect detection of DM are considered. We find that the DM with a mass $m_{X} \gtrsim 4.5 \mathrm{TeV}$ survives in the case of a long-living catalyst. On the other hand, if the catalyst decays during the catalyzed annihilation era, then the bound can be released. We also discuss two paradigms which can maintain the kinetic equilibrium of DM until the DM freeze-out. In both cases, the freeze-out temperature of DM is an order of magnitude higher than the original model.

Keywords: Beyond Standard Model, Gauge Symmetry

ArXiv EPrint: 2107.13475

\footnotetext{
${ }^{1}$ Corresponding author.
} 


\section{Contents}

1 Introduction 1

2 The model 2

$2.1 \mathrm{SU}(2)_{D}$ gauge-Higgs model 2

2.2 Dimension-5 effective operator 6

3 Catalyzed freeze-out of $X_{\mu} \quad 8$

3.1 Annihilation cross sections and decay width 8

$\begin{array}{lll}3.2 & \text { The Boltzmann equations and the solutions } & 10\end{array}$

4 Kinetic equilibrium before DM freeze-out 16

$\begin{array}{ll}4.1 \text { The Higgs-portal strategy } & 16\end{array}$

$\begin{array}{lll}4.2 & \text { The ALP strategy } & 18\end{array}$

5 Conclusion $\quad 20$

\section{Introduction}

Dark Matter (DM) constitutes about $27 \%$ of energy density in the Universe, but its particle properties and production mechanism remain still unknown to us. Observations from cosmology and astrophysics indicate that the DM is mostly likely to be cold when it decouples from the thermal bath. One of the most popular types of cold DM is Weakly Interacting Massive Particles (WIMPs), which are thermally produced in the early Universe and finally frozen out at some temperature $T_{f} \sim m_{D M} / 25$. In this kind of models, DM candidates usually have masses ranging from $1 \mathrm{GeV}$ to $10 \mathrm{TeV}$ and the magnitude of their couplings with SM particles are similar to the weak interaction. Based on these implications, people have designed many experiments to detect WIMPs directly [1-3] and indirectly [4-9].

Recently, a new DM freeze-out paradigm is proposed by Xing and Zhu in ref. [10]. In their setup, the dark sector is nearly secluded, and the depletion of a DM particle $\chi$ is assisted with a catalyst particle $A^{\prime}$, which is slightly lighter than $\chi$. The dominant processes are $2 \chi \rightarrow 2 A^{\prime}$ and $3 A^{\prime} \rightarrow 2 \chi$, in which the yield of $A^{\prime}\left(Y_{A^{\prime}}\right)$ keeps nearly constant until $A^{\prime}$ decays. Note that the model is similar to the secluded DM [11, 12], but the lifetime of the catalyst particle is much longer. They are required to be long-living enough to support the whole catalyzed annihilation processes until the DM freeze-out. In this way, the yield of DM decreases in a manner of $Y_{\chi} \propto x^{-3 / 2}$ during the catalyzed annihilation era. Comparing with the situation of Strongly Interacting Massive Particles (SIMPs) models and their variations [13-17], the depletion efficiency of DMs in the catalyzed annihilation scenario is much slower, and thus the freeze-out temperature is lower. In ref. [10], an U(1)' 
gauge symmetric model with fermionic DM is presented to illustrate how the catalyzed freeze-out mechanism does work. A tiny kinetic mixing between the dark photon and the $\mathrm{U}(1)_{Y}$ gauge field is introduced to enable the catalyst decay.

In this work, we propose a vector DM model in which the DM candidates freeze-out through the catalyzed annihilation. Vector dark matter models has been discussed in many previous studies, such as a U(1) gauge symmetry extension [18-28], a non-abelian gauge symmetry extension [29-42] and a model with non-gauge field vector bosons [43]. We consider an $\mathrm{SU}(2)_{D}$ gauge symmetry which is spontaneously broken by a doublet scalar $\Phi_{D}^{i}$ and a real triplet scalar $\Delta_{D}^{a}$. A complex vector field $X_{\mu} \equiv\left(V_{\mu}^{1}-i V_{\mu}^{2}\right) / \sqrt{2}$, which is formed by two components of the $\mathrm{SU}(2)_{D}$ gauge fields, is regarded as a DM candidate. The remaining gauge field $C_{\mu} \equiv V_{\mu}^{3}$ plays the role of a catalyst. It means that the DM and the catalyst are unified in our model. In order to allow the catalyst to decay, we introduce a dimension-5 effective operator $B^{\mu \nu} \Delta_{D}^{a} V_{\mu \nu}^{a}$ which generates a kinetic mixing term between the catalyst field $C_{\mu}$ and the $\mathrm{U}(1)_{Y}$ gauge field $B_{\mu}$ [44]. This kinetic mixing term can be naturally small since the operator can be suppressed by a large UV completion scale. A condition of catalyzed annihilation is that the catalyst should be lighter than the DM. It is automatically satisfied in our setup since $C_{\mu}$ only acquires mass from the vacuum expectation value (VEV) of the doublet scalar while $X_{\mu}$ acquires mass from both VEVs of the doublet and the triplet. The processes of DMs annihilating into catalysts can lead to significant signals in DM indirect detection experiments, such as the Fermi-LAT [9] and the CTA [45]. We will discuss their constraints and sensitivities in our model.

In the framework of catalyzed freeze-out, a tough problem is raised that the interactions between the dark and the SM sectors are too weak to keep the DM in kinetic equilibrium (KE) with the thermal bath during the catalyzed annihilation era. We propose two template models to alleviate this problem. One is to maintain KE via the DM-fermions scattering mediated by Higgs bosons. It requires a larger Higg-portal coupling comparing to the original model. The other one is to maintain the KE assisted by a thermal axion-like particle (ALP). In both cases, at least one more parameter is needed for determining the freeze-out temperature of the catalyst.

This paper is organized as follows. In section 2, we introduce the $\mathrm{SU}(2)_{D}$ gauge models. In section 3, we discuss the solutions of the Boltzmann equations and some relevant constraints from experiments. In section 4, we discuss two strategies to solve the kinetic equilibrium problem. Finally, we conclude all our findings in the section 5 .

\section{The model}

\section{$2.1 \quad \mathrm{SU}(2)_{D}$ gauge-Higgs model}

In this section, let us present the model. We extend the $\mathrm{SM}$ with an $\mathrm{SU}(2)_{D}$ gauge symmetry which is spontaneously and completely broken by a scalar doublet and a triplet. All the three components of the gauge fields will be massive and two of them are degenerate. The degenerate components can combine to form a complex vector field $X_{\mu}$ (similar to the W boson in the $\mathrm{SM}$ ), which is charged under a global $\mathrm{U}(1)_{D}$ symmetry, while SM particles are neutral. If $X_{\mu}$ is the lightest particle with $\mathrm{U}(1)_{D}$ charge, then it can be a stable DM 
candidate since it does not completely decay into the SM particles. The remaining component of the $\mathrm{SU}(2)_{D}$ gauge fields is a real vector field $C_{\mu}$, which is lighter than $X_{\mu}$ and thus it can play the role of a catalyst.

The Lagrangian of the pure gauge part is

$$
\mathcal{L}_{\text {gauge }}=-\frac{1}{4} V_{\mu \nu}^{a} V^{a \mu \nu}
$$

where $V_{\mu \nu}^{a}=\partial_{\mu} V_{\nu}^{a}-\partial_{\nu} V_{\mu}^{a}+g_{D} \epsilon^{a b c} V_{\mu}^{b} V_{\nu}^{c}$ is the field strength tensor of the $\mathrm{SU}(2)_{D}$ gauge fields $V_{\mu}^{a}(a=1,2,3)$ with gauge coupling $g_{D}$. Let us denote $X_{\mu} \equiv\left(V_{\mu}^{1}-i V_{\mu}^{2}\right) / \sqrt{2}$ and $C_{\mu} \equiv V_{\mu}^{3}$, and rewrite the Lagrangian (2.1) as

$$
\begin{aligned}
\mathcal{L}_{\text {gauge }}= & -\frac{1}{4} C_{\mu \nu} C^{\mu \nu}-\frac{1}{2} \hat{X}_{\mu \nu}^{*} \hat{X}^{\mu \nu}-g_{D}^{2}\left(C_{\mu} C^{\mu} X^{\nu} X_{\nu}^{*}-C_{\mu} C_{\nu} X^{\mu} X^{* \nu}\right) \\
& -\frac{i g_{D}}{2} \hat{X}^{\mu \nu}\left(C_{\mu} X_{\nu}^{*}-C_{\nu} X_{\mu}^{*}\right)+\frac{i g_{D}}{2} \hat{X}^{* \mu \nu}\left(C_{\mu} X_{\nu}-C_{\nu} X_{\mu}\right) \\
& +i g_{D} C^{\mu \nu} X_{\mu} X_{\nu}^{*}-\frac{g_{D}^{2}}{2}\left[\left(X_{\mu}^{*} X^{\mu}\right)^{2}-\left(X_{\mu} X^{\mu}\right)\left(X_{\nu}^{*} X^{* \nu}\right)\right],
\end{aligned}
$$

where $C_{\mu \nu} \equiv \partial_{\mu} C_{\nu}-\partial_{\nu} C_{\mu}$ and $\hat{X}_{\mu \nu} \equiv \partial_{\mu} X_{\nu}-\partial_{\nu} X_{\mu}$. To generate the masses of the vector fields, we introduce an $\mathrm{SU}(2)_{D}$ doublet scalar $\Phi_{D}^{i}=\left(\phi_{1}, \phi_{2}\right)$ and a real triplet scalar $\Delta_{D}^{a}=\left(\Delta_{D}^{1}, \Delta_{D}^{2}, \Delta_{D}^{3}\right)$. The gauge fields couple to the Higgs fields through the covariant derivative terms:

$$
\mathcal{L}_{H}=\left(D_{\mu} \Phi_{D}\right)^{\dagger} D^{\mu} \Phi_{D}+\operatorname{tr}\left[\left(D_{\mu} \Delta_{D}\right)^{\dagger} D^{\mu} \Delta_{D}\right]
$$

where $\Delta_{D} \equiv \Delta_{D}^{a} \sigma^{a} / 2$ with the Pauli matrices $\sigma^{a}$. The covariant derivatives of the scalar fields are given by

$$
\begin{aligned}
D_{\mu} \Phi_{D} & =\left[\partial_{\mu}-i g_{D}\left(\begin{array}{cc}
\frac{C_{\mu}}{2} & \frac{X_{\mu}}{\sqrt{2}} \\
\frac{X_{\mu}^{*}}{\sqrt{2}} & -\frac{C_{\mu}}{2}
\end{array}\right)\right]\left(\begin{array}{c}
\phi_{1} \\
\phi_{2}
\end{array}\right), \\
D_{\mu} \Delta_{D} & =\partial_{\mu}\left(\begin{array}{cc}
\frac{\Delta_{D}^{3}}{2} & \frac{\Delta}{\sqrt{2}} \\
\frac{\Delta^{*}}{\sqrt{2}} & -\frac{\Delta_{D}^{3}}{2}
\end{array}\right)-i g_{D}\left[\left(\begin{array}{cc}
\frac{C_{\mu}}{2} & \frac{X_{\mu}}{\sqrt{2}} \\
\frac{X_{\mu}^{*}}{\sqrt{2}} & -\frac{C_{\mu}}{2}
\end{array}\right),\left(\begin{array}{cc}
\frac{\Delta_{D}^{3}}{2} & \frac{\Delta}{\sqrt{2}} \\
\frac{\Delta^{*}}{\sqrt{2}} & -\frac{\Delta_{D}^{3}}{2}
\end{array}\right)\right],
\end{aligned}
$$

where we have defined a complex scalar field $\Delta \equiv\left(\Delta_{D}^{1}-i \Delta_{D}^{2}\right) / \sqrt{2}$ for convenience. To trigger the spontaneous breaking of $\mathrm{SU}(2)_{D}$, we let the scalar fields acquire non-zero vacuum expectation values (VEVs), and parametrize them as

$$
\Phi_{D}=\left(\begin{array}{c}
\phi_{1} \\
\frac{v_{2}+\varphi+i a}{\sqrt{2}}
\end{array}\right), \quad \Delta_{D}=\left(\begin{array}{cc}
\frac{v_{3}+\rho}{2} & \frac{\Delta}{\sqrt{2}} \\
\frac{\Delta^{*}}{\sqrt{2}} & -\frac{v_{3}+\rho}{2}
\end{array}\right)
$$

where $v_{2} / \sqrt{2}$ and $v_{3}$ are the VEVs of $\phi_{2}$ and $\Delta_{D}^{3}$, respectively. Substituting eq. (2.6) into 
eq. (2.3), we obtain

$$
\begin{aligned}
\mathcal{L}_{H}= & \frac{1}{2}\left(\partial_{\mu} \varphi\right)^{2}+\frac{1}{2}\left(\partial_{\mu} a\right)^{2}+\left(\partial_{\mu} \phi_{1}\right)^{*} \partial^{\mu} \phi_{1}+\frac{1}{2}\left(\partial_{\mu} \rho\right)^{2}+\left(\partial_{\mu} \Delta\right)^{*} \partial^{\mu} \Delta \\
& +\frac{g_{D}}{2} C_{\mu}\left(\varphi \overleftrightarrow{\partial^{\mu}} a+\phi_{1}^{*} i \overleftrightarrow{\partial^{\mu}} \phi_{1}+2 \Delta^{*} i \overleftrightarrow{\partial^{\mu}} \Delta\right) \\
& +\frac{g_{D}}{2} X_{\mu}\left(\phi_{1}^{*} i \partial^{\mu} \varphi-\phi_{1}^{*} \overleftrightarrow{\partial^{\mu}} a-2 \Delta^{*} \overleftrightarrow{\partial^{\mu}} \rho\right)+h . c . \\
& +g_{D}^{2} C_{\mu} C^{\mu}\left[\frac{v_{2}^{2}}{8}+\frac{v_{2}}{4} \varphi+\frac{1}{8} \varphi^{2}+\frac{1}{4}\left|\phi_{1}\right|^{2}+\frac{1}{8} a^{2}+|\Delta|^{2}\right] \\
& +g_{D}^{2} X_{\mu}^{*} X^{\mu}\left[\frac{v_{2}^{2}}{4}+v_{3}^{2}+\frac{v_{2}}{2} \varphi+2 v_{3} \rho+\frac{1}{4} \varphi^{2}+\frac{1}{2}\left|\phi_{1}\right|^{2}+\frac{1}{4} a^{2}+\rho^{2}+|\Delta|^{2}\right] \\
& -g_{D}^{2}\left(v_{3}+\rho\right) X_{\mu}^{*} C^{\mu} \Delta+\text { h.c. }
\end{aligned}
$$

The masses of gauge fields are found to be

$$
m_{C}=\frac{g_{D}}{2} v_{2}, \quad m_{X}=\frac{g_{D}}{2} \sqrt{v_{2}^{2}+4 v_{3}^{2}} \equiv \frac{g_{D}}{2} v_{1},
$$

where we have defined $v_{1} \equiv \sqrt{v_{2}^{2}+4 v_{3}^{2}}$. It is obvious that $X_{\mu}$ is heavier than $C_{\mu}$ due to the contribution from $v_{3}$. If $v_{3} \lesssim 0.56 v_{2}\left(1.5 m_{C} \gtrsim m_{X}\right)$, then the annihilation process $3 C \rightarrow X+X^{*}$ can happen in the non-relativistic limit.

To justify the vacuum configuration, we need to figure out the minimum of the following potential terms of the scalar fields:

$$
\begin{aligned}
V= & -\mu^{2}|H|^{2}+\frac{\lambda}{2}|H|^{4}-\mu_{2}^{2}\left|\Phi_{D}\right|^{2}+\frac{\lambda_{2}}{2}\left|\Phi_{D}\right|^{4}-\mu_{3}^{2} \operatorname{tr}\left[\Delta_{D}^{\dagger} \Delta_{D}\right]+\frac{\lambda_{3}}{2}\left(\operatorname{tr}\left[\Delta_{D}^{\dagger} \Delta_{D}\right]\right)^{2} \\
& +\lambda_{23}\left|\Phi_{D}\right|^{2} \operatorname{tr}\left[\Delta_{D}^{\dagger} \Delta_{D}\right]+\kappa_{23} \Phi_{D}^{\dagger} \Delta_{D} \Phi_{D}+\lambda_{02}|H|^{2}\left|\Phi_{D}\right|^{2}+\lambda_{03}|H|^{2} \operatorname{tr}\left[\Delta_{D}^{\dagger} \Delta_{D}\right]
\end{aligned}
$$

where $H$ is the SM Higgs field parametrized as $H=\left(G^{+},(v+h+i \chi) / \sqrt{2}\right)^{T}$. The extremum conditions of the potential are

$$
\begin{aligned}
{\left[-\mu^{2}+\frac{\lambda}{2} v^{2}+\frac{1}{2}\left(\lambda_{02} v_{2}^{2}+\lambda_{03} v_{3}^{2}\right)\right] v } & =0, \\
{\left[-\mu_{2}^{2}+\frac{\lambda_{2}}{2} v_{2}^{2}+\frac{\lambda_{23}}{2} v_{3}^{2}+\frac{1}{2} \lambda_{02} v^{2}-\frac{\kappa_{23}}{2} v_{3}\right] v_{2} } & =0 \\
{\left[-\mu_{3}^{2}+\frac{\lambda_{3}}{2} v_{3}^{2}+\frac{\lambda_{23}}{2} v_{2}^{2}+\frac{1}{2} \lambda_{03} v^{2}-\frac{\kappa_{23}}{2} \frac{v_{2}^{2}}{v_{3}}\right] v_{3} } & =0 .
\end{aligned}
$$

The mass matrix of the neutral CP-even fields in $(\varphi, \rho, h)$ basis is given by

$$
M_{\text {even }}^{2}=\left(\begin{array}{ccc}
\lambda_{2} v_{2}^{2} & \left(\lambda_{23}-\xi_{23}\right) v_{2} v_{3} & \lambda_{02} v v_{2} \\
\left(\lambda_{23}-\xi_{23}\right) v_{2} v_{3} & \lambda_{3} v_{3}^{2}+\frac{1}{2} \xi_{23} v_{2}^{2} & \lambda_{03} v v_{3} \\
\lambda_{02} v v_{2} & \lambda_{03} v v_{3} & \lambda v^{2}
\end{array}\right)
$$

where $\xi_{23} \equiv \kappa_{23} / 2 v_{3}$. It can be diagonalized by a orthogonal $3 \times 3$ matrix $O$ as follows,

$$
M_{\text {diag }}^{2}=O M_{\text {even }}^{2} O^{T}=\operatorname{diag}\left\{m_{3}^{2}, m_{2}^{2}, m_{1}^{2}\right\} .
$$


We assume $\lambda_{02}$ and $\lambda_{03}$ to be much smaller than $\lambda_{2}$ and $\xi_{23}$ for obtaining a SM-like Higgs boson. The smallness of $\lambda_{02}$ and $\lambda_{03}$ also suppresses the annihilation cross sections of processes, $X^{*}+X \rightarrow \bar{t}+t, W^{+}+W^{-}, Z+Z$, through Higgs-portal. With this assumption, the orthogonal matrix $O$ can now be approximated by

$$
O \approx\left(\begin{array}{ccc}
1 & 0 & -\alpha_{13} \\
0 & 1 & -\alpha_{23} \\
\alpha_{13} & \alpha_{23} & 1
\end{array}\right)\left(\begin{array}{ccc}
c_{\alpha} & -s_{\alpha} & 0 \\
s_{\alpha} & c_{\alpha} & 0 \\
0 & 0 & 1
\end{array}\right)
$$

where $s_{\alpha} \equiv \sin \alpha$ and $c_{\alpha} \equiv \cos \alpha$ and

$$
\begin{aligned}
\tan (2 \alpha) & =\frac{2\left(\lambda_{23}-\xi_{23}\right) v_{2} v_{3}}{\lambda_{2} v_{2}^{2}-\lambda_{3} v_{3}^{2}-\frac{\xi_{23}}{2} v_{2}^{2}}, \\
\alpha_{13} & \approx-\frac{\left(\lambda_{02} v_{2} c_{\alpha}-\lambda_{03} v_{3} s_{\alpha}\right) v}{\lambda_{2} v_{2}^{2} c_{\alpha}^{2}+\left(\lambda_{3} v_{3}^{2}+\frac{\xi_{23}}{2} v_{2}^{2}\right) s_{\alpha}^{2}-\left(\lambda_{23}-\xi_{23}\right) v_{2} v_{3} s_{2 \alpha}-\lambda v^{2}}, \\
\alpha_{23} & \approx-\frac{\left(\lambda_{02} v_{2} s_{\alpha}+\lambda_{03} v_{3} c_{\alpha}\right) v}{\lambda_{2} v_{2}^{2} s_{\alpha}^{2}+\left(\lambda_{3} v_{3}^{2}+\frac{\xi_{23}}{2} v_{2}^{2}\right) c_{\alpha}^{2}+\left(\lambda_{23}-\xi_{23}\right) v_{2} v_{3} s_{2 \alpha}-\lambda v^{2}} .
\end{aligned}
$$

The mass eigenstates and corresponding eigenvalues are given by

$$
\begin{aligned}
\left(\begin{array}{l}
h_{3} \\
h_{2} \\
h_{1}
\end{array}\right) & =O\left(\begin{array}{l}
\varphi \\
\rho \\
h
\end{array}\right) \\
m_{3}^{2} & \approx \lambda_{2} v_{2}^{2} c_{\alpha}^{2}+\left(\lambda_{3} v_{3}^{2}+\frac{\xi_{23}}{2} v_{2}^{2}\right) s_{\alpha}^{2}-\left(\lambda_{23}-\xi_{23}\right) v_{2} v_{3} s_{2 \alpha} \\
m_{2}^{2} & \approx \lambda_{2} v_{2}^{2} s_{\alpha}^{2}+\left(\lambda_{3} v_{3}^{2}+\frac{\xi_{23}}{2} v_{2}^{2}\right) c_{\alpha}^{2}+\left(\lambda_{23}-\xi_{23}\right) v_{2} v_{3} s_{2 \alpha} \\
m_{1}^{2} & \approx \lambda v^{2}
\end{aligned}
$$

We will assume that $m_{1} \approx 126 \mathrm{GeV}$ is the mass of SM-like Higgs boson in later discussion. The CP-odd scalar $a$ is a Goldstone boson eaten by the gauge field $C_{\mu}$. The mass matrix of the complex scalar $\left(\phi_{1}, \Delta\right)$ is

$$
M_{c}^{2}=\frac{1}{2} \xi_{23}\left(\begin{array}{cc}
4 v_{3}^{2} & 2 v_{2} v_{3} \\
2 v_{2} v_{3} & v_{2}^{2}
\end{array}\right),
$$

which can be diagonalized by a rotation

$$
R_{\theta} M_{c}^{2} R_{\theta}^{T}=\left(\begin{array}{cc}
0 & 0 \\
0 & m_{s}^{2}
\end{array}\right), \quad R_{\theta}=\left(\begin{array}{cc}
c_{\theta} & -s_{\theta} \\
s_{\theta} & c_{\theta}
\end{array}\right)
$$

where

$$
s_{\theta} \equiv \sin \theta=\frac{2 v_{3}}{v_{1}}, \quad c_{\theta} \equiv \cos \theta=\frac{v_{2}}{v_{1}}, \quad m_{s}^{2}=\xi_{23} v_{1}^{2} .
$$




\subsection{Dimension-5 effective operator}

We can check that $(2.2),(2.7)$, and (2.9) are invariant under a global $\mathrm{U}(1)_{D}$ transformation:

$$
\Delta \rightarrow e^{i \gamma} \Delta, \quad \phi_{1} \rightarrow e^{i \gamma} \phi_{1}, \quad X_{\mu} \rightarrow e^{i \gamma} X_{\mu} .
$$

Therefore, $X_{\mu}$ cannot decay if it is lighter than $\Delta$ and $\phi_{1}$. In addition, there is a discrete symmetry $G_{D}$ in the $\mathrm{SU}(2)_{D}$ gauge and Higgs sector. We can check that (2.2), (2.3) and (2.9) are invariant under the following $G_{D}$ transformations

$$
C_{\mu} \rightarrow-C_{\mu}, \quad X_{\mu} \rightarrow X_{\mu}^{*}, \quad \phi_{1} \rightarrow-\phi_{1}^{*}, \quad \phi_{2} \rightarrow \phi_{2}^{*} \quad \Delta \rightarrow-\Delta^{*}, \quad \Delta_{D}^{3} \rightarrow \Delta_{D}^{3} .
$$

This symmetry is preserved even after the $\phi_{2}$ and $\Delta_{D}^{3}$ acquiring VEVs. If we assume that the gauge fields $C_{\mu}$ and $X_{\mu}$ are much lighter than the Higgs fields $\Delta_{D}$ and $\Phi_{D}$, then the lightest particle in this sector is $C_{\mu}$ and it can not decay either due to the $G_{D}$ symmetry. According to the requirement of the catalyzed freeze-out mechanism, the catalyst $C_{\mu}$ should be long-living but unstable, so we need to add something new to slightly violate $G_{D}$. As an effective theory in low energy, we can introduce a dimension-5 operator:

$$
\mathcal{L}_{5}=-\frac{c}{\Lambda} B^{\mu \nu} \Delta_{D}^{a} V_{\mu \nu}^{a},
$$

where $c$ is a Wilson coefficient, and $\Lambda$ is some UV complete scale. We can check that $\Delta_{D}^{a} V_{\mu \nu}^{a} \rightarrow-\Delta_{D}^{a} V_{\mu \nu}^{a}$ under the $G_{D}$ transformation, and thus $\mathcal{L}_{5}$ violates the symmetry. Substituting (2.6) into the operator (2.28), we find it includes the following terms,

$$
\mathcal{L}_{5} \supset-\frac{c}{\Lambda} B^{\mu \nu} \Delta_{D}^{3} V_{\mu \nu}^{3}=-\frac{c\left(v_{3}+\rho\right)}{\Lambda} B^{\mu \nu} C_{\mu \nu}+\frac{i g_{D} c\left(v_{3}+\rho\right)}{\Lambda} B^{\mu \nu}\left(X_{\mu} X_{\nu}^{*}-X_{\mu}^{*} X_{\nu}\right) .
$$

The first term is an effective kinetic mixing between $B_{\mu}$ and $C_{\mu}$ fields, while the second term includes an electromagnetic interaction of the magnetic moment of $X_{\mu} \cdot{ }^{1}$ Due to the kinetic mixing, the $C_{\mu}$ can finally decay into SM particles.

Note that the kinetic terms of $B_{\mu}$ and $C_{\mu}$ are not in the canonical form, so we should figure out a new basis $\left(\hat{B}_{\mu}, \hat{C}_{\mu}\right)$ such that all fields are canonically normalized. It can be done by the following transformation of basis [46-48]:

$$
\left(\begin{array}{l}
B_{\mu} \\
C_{\mu}
\end{array}\right)=\left(\begin{array}{cc}
1 & -t_{\epsilon} \\
0 & \frac{1}{c_{\epsilon}}
\end{array}\right)\left(\begin{array}{c}
\hat{B}_{\mu} \\
\hat{C}_{\mu}
\end{array}\right)
$$

where $s_{\epsilon} \equiv 2 c v_{3} / \Lambda$. The interaction part of the effective operator in terms of $\left(\hat{B}_{\mu}, \hat{C}_{\mu}\right)$ is given by

$$
\begin{aligned}
\mathcal{L}_{5} \supset & -\frac{t_{\epsilon}}{2 v_{3}} \rho \hat{B}^{\mu \nu} \hat{C}_{\mu \nu}+\frac{i g_{D} s_{\epsilon}}{2 v_{3}} \rho \hat{B}^{\mu \nu}\left(X_{\mu} X_{\nu}^{*}-X_{\mu}^{*} X_{\nu}\right) \\
& -\frac{s_{\epsilon}}{2 v_{3}} \hat{B}^{\mu \nu}\left[\Delta\left(\hat{X}_{\mu \nu}^{*}+\frac{i g_{D}}{c_{\epsilon}}\left(\hat{C}_{\mu} X_{\nu}^{*}-\hat{C}_{\nu} X_{\mu}^{*}\right)\right)+\text { h.c. }\right]+\ldots,
\end{aligned}
$$

\footnotetext{
${ }^{1}$ In ref. [40], the electric and magnetic multipole moments of vector DM are studied in details.
} 
where we have neglected $\mathcal{O}\left(s_{\epsilon}^{2}\right)$ and other higher order terms. In the new basis, the covariant derivatives of the scalar fields are given by

$$
\begin{aligned}
D_{\mu} H & =\left[\partial_{\mu}-i g W_{\mu}^{a} \frac{\tau^{a}}{2}-i \frac{g^{\prime}}{2} \hat{B}_{\mu}+\frac{i g^{\prime} t_{\epsilon}}{2} \hat{C}_{\mu}\right] H \\
D_{\mu} \Phi_{D} & =\partial_{\mu} \Phi_{D}-i g_{D}\left(\begin{array}{cc}
\frac{\hat{C}_{\mu}}{2 c_{\epsilon}} & \frac{X_{\mu}}{\sqrt{2}} \\
\frac{X_{\mu}^{*}}{\sqrt{2}}-\frac{\hat{C}_{\mu}}{2 c_{\epsilon}}
\end{array}\right) \Phi_{D} \\
D_{\mu} \Delta_{D} & =\partial_{\mu} \Delta_{D}-i g_{D}\left[\left(\begin{array}{cc}
\frac{\hat{C}_{\mu}}{2 c_{\epsilon}} & \frac{X_{\mu}}{\sqrt{2}} \\
\frac{X_{\mu}^{*}}{\sqrt{2}} & -\frac{\hat{C}_{\mu}}{2 c_{\epsilon}}
\end{array}\right) \Delta_{D}-\Delta_{D}\left(\begin{array}{cc}
\frac{\hat{C}_{\mu}}{2 c_{\epsilon}} & \frac{X_{\mu}}{\sqrt{2}} \\
\frac{X_{\mu}^{*}}{\sqrt{2}} & -\frac{\hat{C}_{\mu}}{2 c_{\epsilon}}
\end{array}\right)\right] .
\end{aligned}
$$

The masses of $W_{\mu}^{ \pm}, X_{\mu}$ and the neutral gauge fields $\left(W_{\mu}^{3}, \hat{B}_{\mu}, \hat{C}_{\mu}\right)$ can be read off as follows,

$$
\begin{aligned}
m_{W}^{2} & =\frac{g^{2}}{4} v^{2}, \quad m_{X}^{2}=g_{D}^{2}\left(\frac{v_{2}^{2}}{4}+v_{3}^{2}\right), \\
M_{g}^{2} & =\frac{1}{4}\left(\begin{array}{ccc}
g^{2} v^{2} & -g g^{\prime} v^{2} & g g^{\prime} t_{\epsilon} v^{2} \\
-g g^{\prime} v^{2} & g^{\prime 2} v^{2} & -g^{\prime 2} t_{\epsilon} v^{2} \\
g g^{\prime} t_{\epsilon} v^{2} & -g^{\prime 2} t_{\epsilon} v^{2} & g^{\prime 2} t_{\epsilon}^{2} v^{2}+g_{D}^{2} v_{2}^{2}
\end{array}\right) .
\end{aligned}
$$

$M_{g}^{2}$ can be diagonalized by an orthogonal transformation $m_{g}^{2}=O_{g} M_{g}^{2} O_{g}^{T}=$ $\operatorname{diag}\left\{0, m_{Z}^{2}, m_{Z^{\prime}}^{2}\right\}$, where

$$
\begin{aligned}
O_{g} & =\left(\begin{array}{ccc}
1 & 0 & 0 \\
0 & c_{\zeta} & -s_{\zeta} \\
0 & s_{\zeta} & c_{\zeta}
\end{array}\right)\left(\begin{array}{ccc}
\hat{s}_{W} & \hat{c}_{W} & 0 \\
\hat{c}_{W} & -\hat{s}_{W} & 0 \\
0 & 0 & 1
\end{array}\right), \\
\tan (2 \zeta) & =\frac{2 s_{\epsilon} c_{\epsilon} \hat{s}_{W}\left(g^{2}+g^{\prime 2}\right) v^{2}}{\left(g^{2}+g^{\prime 2}\right) v^{2} c_{\epsilon}^{2}\left(1-\hat{s}_{W}^{2} t_{\epsilon}^{2}\right)-g_{D}^{2} v_{2}^{2}}, \\
m_{Z}^{2} & =\frac{\left(g^{2}+g^{\prime 2}\right)}{4}\left(1+\hat{s}_{W} t_{\epsilon} t_{\zeta}\right), \quad m_{Z^{\prime}}^{2}=\frac{g_{D}^{2} v_{2}^{2}}{4 c_{\epsilon}^{2}\left(1+\hat{s}_{W} t_{\epsilon} t_{\zeta}\right)},
\end{aligned}
$$

and $\hat{s}_{W} \equiv \sin \hat{\theta}_{W}=g^{\prime} / \sqrt{g^{2}+g^{\prime 2}}$ is the sine of the Weinberg angle. When $s_{\epsilon} \ll 1$ and $\sqrt{g^{2}+g^{\prime 2}} v \ll g_{D} v_{2}, t_{\zeta} \equiv \tan \xi$ can be approximated by

$$
t_{\zeta} \approx \frac{\hat{s}_{W} t_{\epsilon}}{1-r}
$$

where $r \equiv m_{Z^{\prime}}^{2} / m_{Z}^{2}$. The mass eigenstate $Z_{\mu}^{\prime}$ is the true catalyst particle and it is very closed to the gauge eigenstate $C_{\mu}$ in the $s_{\epsilon} \ll 1$ limit. For discussing the phenomenologies later, we show the SM neutral current interactions in terms of gauge fields mass eigenstates as follows,

$$
\begin{aligned}
\mathcal{L}_{N C}= & e J_{E M}^{\mu} A_{\mu}+\left[\frac{g}{2 \hat{c}_{W}}\left(\hat{s}_{W} s_{\zeta} t_{\epsilon}+c_{\zeta}\right) J_{Z}^{\mu}-e \hat{c}_{W} s_{\zeta} t_{\epsilon} J_{E M}^{\mu}\right] Z_{\mu} \\
& +\left[\frac{g}{2 \hat{c}_{W}}\left(\hat{s}_{W} c_{\zeta} t_{\epsilon}-s_{\zeta}\right) J_{Z}^{\mu}-e \hat{c}_{W} c_{\zeta} t_{\epsilon} J_{E M}^{\mu}\right] Z_{\mu}^{\prime},
\end{aligned}
$$

where $J_{E M}^{\mu}$ and $J_{Z}^{\mu}$ correspond to the neutral currents of SM fermions. ${ }^{2}$

\footnotetext{
${ }^{2}$ More details can be found in ref. [48].
} 
Finally, we want to point out that a possible UV completion of the operator (2.28) is to introduce a super heavy vector-like fermion $\Psi=\left(\Psi_{1}, \Psi_{2}\right)^{T}$ which is a doublet of $\mathrm{SU}(2)_{D}$ with hypercharge $Y=-1$. The Lagrangian of $\Psi$ is given by

$$
\mathcal{L}_{\Psi}=\bar{\Psi}\left(i \not D-m_{\Psi}\right) \Psi-y_{3} \bar{\Psi} \Delta_{D} \Psi-y_{2}^{I} \bar{\Psi} \Phi_{D} e_{R}^{I}+\text { h.c. },
$$

where $e_{R}^{I}$ is the $I$-th generation of right-handed charged lepton. When $y_{3}=y_{2}^{I}=0$, the $G_{D}$ symmetry is respected if $\Psi$ transforms in the following way

$$
\Psi_{1} \leftrightarrow \Psi_{2}
$$

Once $y_{3}$ and $y_{2}^{I}$ are turned on, the $G_{D}$ symmetry is broken and then the operator (2.28) can be induced by loops of $\Psi_{1,2}$. Using the formula given in ref. [40], the mixing parameter is

$$
s_{\epsilon} \sim \frac{g_{D} g^{\prime}}{6 \pi^{2}}\left(\frac{y_{3} v_{3}}{m_{\Psi}}\right) .
$$

For the purpose of obtaining a value $t_{\epsilon} \sim 10^{-11}$, we need to set $m_{\Psi} \sim 10^{12} \mathrm{GeV}$ when $v_{3} \sim 1 \mathrm{TeV}$.

\section{Catalyzed freeze-out of $X_{\mu}$}

\subsection{Annihilation cross sections and decay width}

The dominant annihilation processes of DM pairs to SM particles are $X^{*}+X \rightarrow W^{+}+$ $W^{-}, Z+Z, h+h, \bar{f}+f$ through s-channel mediated by Higgs bosons and gauge bosons. Since the annihilation cross sections of gauge boson portal processes are suppressed by $s_{\epsilon}^{2} \sim$ $v_{3}^{2} / \Lambda^{2}$ which is assumed to be extremely small, we only need to compute the contributions from Higgs-portal processes. The corresponding annihilation cross sections are given by

$$
\begin{aligned}
& \langle\sigma v\rangle_{X^{*} X \rightarrow 2 \phi_{\mathrm{SM}}} \approx\langle\sigma v\rangle_{X^{*} X \rightarrow \bar{t} t}+\langle\sigma v\rangle_{X^{*} X \rightarrow W^{+} W^{-}}+\langle\sigma v\rangle_{X^{*} X \rightarrow Z Z}+\langle\sigma v\rangle_{X^{*} X \rightarrow h_{1} h_{1}} \\
& \langle\sigma v\rangle_{X^{*} X \rightarrow \bar{t} t} \approx \frac{g_{D}^{4} v_{2}^{2}}{256 \pi m_{X}^{2} v^{2}}\left(\frac{m_{t}^{2}}{m_{X}^{2}}\right)\left[\left(c_{\alpha} \alpha_{13}+s_{\alpha} \alpha_{23}\right)+\frac{4 v_{3}}{v_{2}}\left(c_{\alpha} \alpha_{23}-s_{\alpha} \alpha_{13}\right)\right]^{2} \\
& \langle\sigma v\rangle_{X^{*} X \rightarrow V V} \approx \frac{g_{D}^{4} v_{2}^{2}}{256 \pi m_{X}^{2} v^{2}}\left[\left(c_{\alpha} \alpha_{13}+s_{\alpha} \alpha_{23}\right)+\frac{4 v_{3}}{v_{2}}\left(c_{\alpha} \alpha_{23}-s_{\alpha} \alpha_{13}\right)\right]^{2} \\
& \langle\sigma v\rangle_{X^{*} X \rightarrow h_{1} h_{1}} \approx\left(\frac{1}{3}\right) \frac{g_{D}^{4} v_{2}^{2}}{256 \pi m_{X}^{2} v^{2}}\left[\alpha_{13} c_{2 \alpha}+\left(\frac{m_{2}^{2}}{m_{3}^{2}}\right) \alpha_{23} s_{2 \alpha}-\frac{4 v_{3}}{v_{2}}\left(\alpha_{13} s_{2 \alpha}-\left(\frac{m_{2}^{2}}{m_{3}^{2}}\right) \alpha_{23} c_{2 \alpha}\right)\right]^{2} \\
& \langle\sigma v\rangle_{Z^{\prime} Z^{\prime} \rightarrow 2 \phi_{\mathrm{SM}}} \approx\langle\sigma v\rangle_{Z^{\prime} Z^{\prime} \rightarrow \overline{t t}}+\langle\sigma v\rangle_{Z^{\prime} Z^{\prime} \rightarrow W^{+} W^{-}}+\langle\sigma v\rangle_{Z^{\prime} Z^{\prime} \rightarrow Z Z}+\langle\sigma v\rangle_{Z^{\prime} Z^{\prime} \rightarrow h_{1} h_{1}}, \\
& \langle\sigma v\rangle_{Z^{\prime} Z^{\prime} \rightarrow \bar{t} t} \approx \frac{g_{D}^{4} v_{2}^{2}}{512 \pi m_{Z^{\prime}}^{2} v^{2}}\left(\frac{m_{t}^{2}}{m_{Z^{\prime}}^{2}}\right)\left(c_{\alpha} \alpha_{13}+s_{\alpha} \alpha_{23}\right)^{2} \\
& \langle\sigma v\rangle_{Z^{\prime} Z^{\prime} \rightarrow V V} \approx \frac{g_{D}^{4} v_{2}^{2}}{512 \pi m_{Z^{\prime}}^{2} v^{2}}\left(c_{\alpha} \alpha_{13}+s_{\alpha} \alpha_{23}\right)^{2} \\
& \langle\sigma v\rangle_{Z^{\prime} Z^{\prime} \rightarrow h_{1} h_{1}} \approx\left(\frac{1}{3}\right) \frac{g_{D}^{4} v_{2}^{2}}{256 \pi m_{Z^{\prime}}^{2} v^{2}}\left[\alpha_{13} c_{2 \alpha}+\left(\frac{m_{2}^{2}}{m_{3}^{2}}\right) \alpha_{23} s_{2 \alpha}\right]^{2}
\end{aligned}
$$



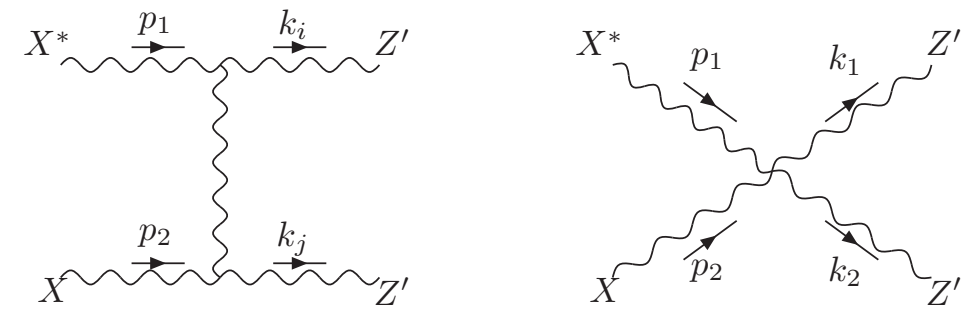

Figure 1. Feynman diagrams of $X^{*}+X \rightarrow Z^{\prime}+Z^{\prime}$ processes. There are 2 independent diagrams with $i, j=1,2$ and $i \neq j$ for the first plot.

where $2 \phi_{\mathrm{SM}}$ represents all dominant SM final states including $\bar{t} t, W^{+} W^{-}, Z Z, h_{1} h_{1}$, while $V V$ represents two vector bosons $Z+Z, W^{+}+W^{-}$. For simplicity, we have assumed that the model parameters satisfy $m_{t}^{2}, m_{1}^{2}, m_{W}^{2}, m_{Z}^{2} \ll m_{X}^{2}$ and $m_{X}^{2}, m_{Z^{\prime}}^{2} \ll m_{2}^{2}, m_{3}^{2}$. All these annihilation cross sections are suppressed by the couplings, $\lambda_{02}$ and $\lambda_{03}$, and hence we can further assume $\lambda_{02}$ and $\lambda_{03}$ are very small such that $Z^{\prime}+Z^{\prime}\left(X^{*}+X\right) \rightarrow h_{i} \rightarrow 2 \phi_{\text {SM }}$ processes fall behind the Hubble expansion early $(x \sim 10)$. Note that very small $\lambda_{02}$ and $\lambda_{03}$ also suppress the Higgs-portal DM-nuclei scattering cross section, and thus the model can easily circumvent the stringent direct detection bound. However, a very weak coupling is hard to keep DM in kinetic equilibrium with the thermal bath until freeze-out. At the moment, we just assume that the kinetic equilibrium is maintained by some unknown mechanisms. In the next section, we will introduce two strategies for solving this problem, but we need to pay the price that one more parameter is needed for determining the relic abundance of DM.

The annihilation cross section of $X^{*}+X \rightarrow Z^{\prime}+Z^{\prime}$ process is neither suppressed by the kinetic mixing parameter $s_{\epsilon}$ nor the Higgs mixing couplings $\lambda_{02}, \lambda_{03}$. In the situation of $m_{X}^{2} \ll m_{2}^{2}, m_{3}^{2}$, the dominant diagrams of the process are shown in figure 1 , and the annihilation cross section to the leading order is

$$
\left\langle\sigma_{2} v\right\rangle \approx \frac{g_{D}^{4}\left(1-r_{X Z^{\prime}}^{-1}\right)^{1 / 2}\left(152 r_{X Z^{\prime}}^{4}-136 r_{X Z^{\prime}}^{3}+128 r_{X Z^{\prime}}^{2}-18 r_{X Z^{\prime}}+3\right)}{144 \pi m_{Z^{\prime}}^{2} r_{X Z^{\prime}}^{3}\left(2 r_{X Z^{\prime}}-1\right)^{2}}
$$

where $r_{X Z^{\prime}}=m_{X}^{2} / m_{Z^{\prime}}^{2} \approx c_{\theta}^{-2}$.

The catalyzed freeze-out production of DM also requires a $Z^{\prime}+Z^{\prime}+Z^{\prime} \rightarrow X^{*}+X$ process whose reaction rate is comparable with the $X^{*}+X \rightarrow Z^{\prime}+Z^{\prime}$ process during the catalyzed annihilation stage. The diagrams of $Z^{\prime}+Z^{\prime}+Z^{\prime} \rightarrow X^{*}+X$ are shown in figure 2 . Once the amplitude is written down, the corresponding annihilation cross section can be computed in the non-relativistic limit by using the formula (E4) in ref. [49], ${ }^{3}$ and the result

\footnotetext{
${ }^{3}$ In our definition, the annihilation cross section is $1 / S_{i}$ times of the one defined in ref. [49], where $S_{i}=n_{i}$ is a symmetry factor from identical initial particles.
} 

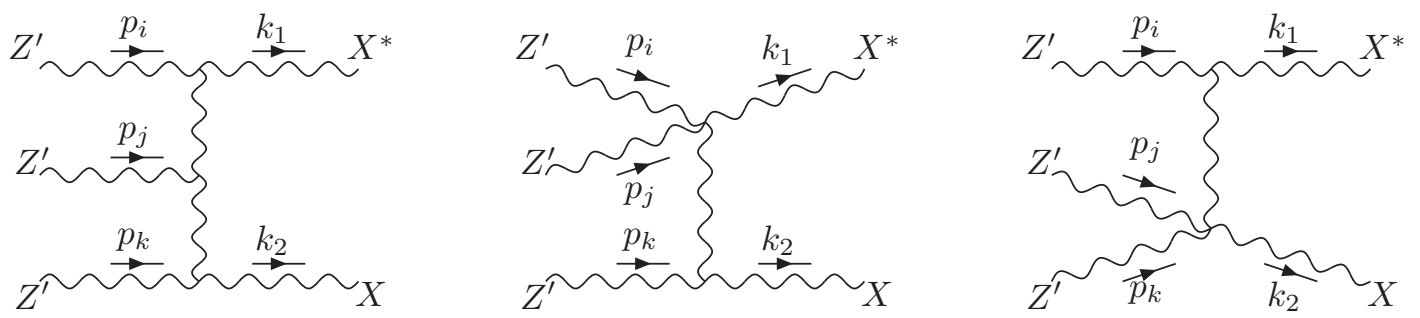

Figure 2. Feynman diagrams of $Z^{\prime}+Z^{\prime}+Z^{\prime} \rightarrow X^{*}+X$ processes. There are 6 independent diagrams with $i, j, k=1,2,3$ and $i \neq j \neq k$ for the first plot, while 3 independent diagrams each for the second and third plots.

is given by

$$
\begin{aligned}
\left\langle\sigma_{3} v^{2}\right\rangle \approx & \frac{1}{6} \frac{g_{D}^{6}}{192 \pi m_{Z^{\prime}}^{5}}\left(1-\frac{4}{9} r_{X Z^{\prime}}\right)^{1 / 2} f\left(r_{X Z^{\prime}}\right), \\
f\left(r_{X Z^{\prime}}\right)= & \frac{729}{256} r_{X Z^{\prime}}^{-6}-\frac{243}{16} r_{X Z^{\prime}}^{-5}+\frac{675}{16} r_{X Z^{\prime}}^{-4}+\frac{1285}{8} r_{X Z^{\prime}}^{-3}-\frac{1007}{4} r_{X Z^{\prime}}^{-2}+\frac{2585}{4} r_{X Z^{\prime}}^{-1} \\
& -\frac{2317}{4}+415 r_{X Z^{\prime}}-12 r_{X Z^{\prime}}^{2}-48 r_{X Z^{\prime}}^{3} .
\end{aligned}
$$

Finally, we need to figure out the decay width of the catalyst. As we have discussed in previous section, catalyst decay due to the dim- $5 G_{D}$ violated operator (2.28) and thus the decay width must be suppressed by $s_{\epsilon}^{2}$. The two-body decay processes are $Z^{\prime} \rightarrow$ $\bar{f}+f, W^{+}+W^{-}$, where $f$ indicates all type of SM fermions. In the $m_{f}, m_{W} \ll m_{Z^{\prime}}$ limit, the total width can be approximately evaluated as $^{4}$

$$
\Gamma_{Z^{\prime}} \approx \frac{27 \alpha t_{\epsilon}^{2} c_{\zeta}^{2} m_{Z^{\prime}}}{16 \hat{c}_{W}^{2}} \approx 2 \times 10^{-2} \times t_{\epsilon}^{2} m_{Z^{\prime}}
$$

\subsection{The Boltzmann equations and the solutions}

The Boltzmann equations of the $X$ and $Z^{\prime}$ read,

$$
\begin{aligned}
\frac{d n_{X}}{d t}+3 H n_{X} \approx & -\frac{1}{2}\langle\sigma v\rangle_{X^{*} X \rightarrow 2 \phi_{\mathrm{SM}}}\left(n_{X}^{2}-\bar{n}_{X}^{2}\right) \\
& -\frac{1}{2}\left\langle\sigma_{2} v\right\rangle\left(n_{X}^{2}-\bar{n}_{X}^{2} \frac{n_{Z^{\prime}}^{2}}{\bar{n}_{Z^{\prime}}^{2}}\right)+2\left\langle\sigma_{3} v^{2}\right\rangle\left(n_{Z^{\prime}}^{3}-\bar{n}_{Z^{\prime}}^{3} \frac{n_{X}^{2}}{\bar{n}_{X}^{2}}\right) \\
\frac{d n_{Z^{\prime}}}{d t}+3 H n_{Z^{\prime}} \approx & -2\langle\sigma v\rangle_{Z^{\prime} Z^{\prime} \rightarrow 2 \phi_{\mathrm{SM}}}\left(n_{Z^{\prime}}^{2}-\bar{n}_{Z^{\prime}}^{2}\right)-\left\langle\Gamma_{Z^{\prime}}\right\rangle\left(n_{Z^{\prime}}-\bar{n}_{Z^{\prime}}\right) \\
& +\frac{1}{2}\left\langle\sigma_{2} v\right\rangle\left(n_{X}^{2}-\bar{n}_{X}^{2} \frac{n_{Z^{\prime}}^{2}}{\bar{n}_{Z^{\prime}}^{2}}\right)-3\left\langle\sigma_{3} v^{2}\right\rangle\left(n_{Z^{\prime}}^{3}-\bar{n}_{Z^{\prime}}^{3} \frac{n}{X}_{\bar{n}_{X}^{2}}^{2}\right)
\end{aligned}
$$

where $\bar{n}_{i}$ is the equilibrium distribution of particle species $i .^{5}$ In a very early era, the Higgs-portal interactions between the dark and SM sectors can thermalize both $X$ and $Z^{\prime}$.

\footnotetext{
${ }^{4}$ The width of $Z^{\prime}$ in our model is the same as the one given in ref. [50].

${ }^{5}$ Note that $n_{X}$ is defined as the sum of DM and anti-DM densities.
} 
Their number density distributions trace the standard Boltzmann distribution:

$$
n_{X} \approx \bar{n}_{X} \approx 2 \times 3 \times\left(\frac{m_{X} T}{2 \pi}\right)^{3 / 2} e^{-m_{X} / T}, \quad n_{Z^{\prime}} \approx \bar{n}_{Z^{\prime}} \approx 3 \times\left(\frac{m_{Z^{\prime}} T}{2 \pi}\right)^{3 / 2} e^{-m_{Z^{\prime}} / T},
$$

where we have assume that the chemical potentials are negligible. Since there are many parameters involved in the annihilation processes, for simplicity, we parametrize the total annihilation cross sections of $X^{*}+X\left(Z^{\prime}+Z^{\prime}\right) \rightarrow \phi_{\mathrm{SM}}+\phi_{\mathrm{SM}}$ as

$$
\begin{aligned}
\langle\sigma v\rangle_{X^{*} X \rightarrow 2 \phi_{\mathrm{SM}}} & =\frac{g_{D}^{4} v_{2}^{2}}{256 \pi m_{X}^{2} v^{2}}\left(\frac{m_{t}^{2}}{m_{X}^{2}}\right) \xi_{X}^{2}, \\
\langle\sigma v\rangle_{Z^{\prime} Z^{\prime} \rightarrow 2 \phi_{\mathrm{SM}}} & =\frac{g_{D}^{4} v_{2}^{2}}{512 \pi m_{Z^{\prime}}^{2} v^{2}}\left(\frac{m_{t}^{2}}{m_{Z^{\prime}}^{2}}\right) \xi_{Z^{\prime}}^{2},
\end{aligned}
$$

where $\xi_{X}$ and $\xi_{Z^{\prime}}$ encode the effects of all the Higgs-portal channels and relevant parameters, e.g. $\alpha, \alpha_{13}, \alpha_{23}$. As the temperature decreases, these processes fall behind the Hubble expansion and we assume that it happens before $T \sim m_{X} / 10$. After that, the first terms in the right-handed sides of Boltzmann equations for both $X$ and $Z^{\prime}$ can be dropped. At the moment, let us assume that the decay and inverse decay terms of $Z^{\prime}$ are negligible before the DM freeze-out and thus the second term in the right-handed side of the Boltzmann equation for $Z^{\prime}$ can be dropped too.

DM and catalyst still keep in thermal equilibrium since both $X^{*}+X \leftrightarrow Z^{\prime}+Z^{\prime}$ and $Z^{\prime}+Z^{\prime}+Z^{\prime} \leftrightarrow X^{*}+X$ reactions are efficient enough for forcing the distributions to satisfy

$$
\frac{n_{X}}{\bar{n}_{X}} \approx \frac{n_{Z^{\prime}}}{\bar{n}_{Z^{\prime}}}, \quad\left(\frac{n_{X}}{\bar{n}_{X}}\right)^{2} \approx\left(\frac{n_{Z^{\prime}}}{\bar{n}_{Z^{\prime}}}\right)^{3} .
$$

The only reasonable solutions to these equations are $n_{X}=\bar{n}_{X}, n_{Z^{\prime}}=\bar{n}_{Z^{\prime}}$.

To figure out the temperature of departure from chemical equilibrium, we can firstly sum up the two equations, (3.6) and (3.7), and get

$$
\frac{d\left(n_{Z^{\prime}}+n_{X}\right)}{d t}+3 H\left(n_{Z^{\prime}}+n_{X}\right) \approx-\left\langle\sigma_{3} v^{2}\right\rangle\left(n_{Z^{\prime}}^{3}-\bar{n}_{Z^{\prime}}^{3} \frac{n_{X}^{2}}{\bar{n}_{X}^{2}}\right) .
$$

Since $X$ is heavier than $Z^{\prime}$ and thus $n_{X} \approx \bar{n}_{X} \ll \bar{n}_{Z^{\prime}}$, we can neglect the $n_{X}$ in the left-handed side of the equation. The evolution of $n_{Z^{\prime}}$ is now determined only by the competition between $\left\langle\sigma_{3} v^{2}\right\rangle$ term and the Hubble expansion. We can expect that $Z^{\prime}$ freezes out when

$$
\left\langle\sigma_{3} v^{2}\right\rangle n_{Z^{\prime}}^{3} \simeq H n_{Z^{\prime}}
$$

To determine the departure temperature $T_{c}$ (or $x_{c} \equiv m_{X} / T_{c}$ ) more precisely, we define $n_{Z^{\prime}} \equiv \bar{n}_{Z^{\prime}}(1+\delta(x))$ and introduce $x \equiv m_{X} / T, Y_{Z^{\prime}} \equiv n_{Z^{\prime}} / s$, where $s=\left(2 \pi^{2} / 45\right) g_{*} T^{3}$ is the entropy density. ${ }^{6}$ Note that $\left\langle\sigma_{2} v^{2}\right\rangle n_{X}^{2}$ is still much larger than $H n_{X}$ at $T=T_{c}$, so $n_{X}$

\footnotetext{
${ }^{6}$ In this work, since the temperature we consider is above several $\mathrm{GeV}$, we make an approximation that $g_{* s} \approx g_{*}$ for simplicity.
} 
is forced to satisfy $n_{X} / \bar{n}_{X}=n_{Z^{\prime}} / \bar{n}_{Z^{\prime}}$. Using the relation $Y_{X} / \bar{Y}_{X}=Y_{Z^{\prime}} / \bar{Y}_{Z^{\prime}}=1+\delta$, the Boltzmann equation of $Z^{\prime}$ becomes

$$
\frac{d \ln \bar{Y}_{Z^{\prime}}}{d x}(1+\delta)+\frac{d \delta}{d x} \approx-\frac{\lambda_{X}}{x^{2}}\left\langle\sigma_{3} v^{2}\right\rangle s \bar{Y}_{Z^{\prime}}^{2}(1+\delta)^{2} \delta,
$$

where $\lambda_{X} \equiv \sqrt{\pi g_{*} / 45} m_{X} m_{p l}$. Since $Y_{Z^{\prime}}$ closely trace the equilibrium distribution, $d \delta / d x$ term is negligible before $Y_{Z^{\prime}}$ frozen. We can take a reference quantity $\delta_{c} \equiv \delta\left(x_{c}\right) \sim 2.5$ as a sign of $Z^{\prime}$ starting departure from thermal equilibrium, then $x_{c}$ can be approximately determined by

$$
x_{c}=r_{X Z^{\prime}}^{1 / 2} W_{0}(\sqrt{A}), \quad A \equiv \frac{9 \lambda_{X}\left\langle\sigma_{3} v^{2}\right\rangle m_{X}^{3}\left(1+\delta_{c}\right) \delta_{c}}{(2 \pi)^{5} r_{X Z^{\prime}}^{2}\left(1-\frac{3 r_{X Z^{\prime}}^{1 / 2}}{2 x_{a}}\right)}\left(\frac{90}{g_{*}}\right),
$$

where $x_{a} \sim 16$ is an approximate value of $x_{c}$ coming from the first iteration of (3.15) and $W_{0}(z)$ is the principle branch of Lambert $W$ function. After $T \gtrsim T_{c}, n_{Z^{\prime}}$ starts to deviate from the Boltzmann suppressed equilibrium distribution, and thus $Y_{Z^{\prime}}>\bar{Y}_{Z^{\prime}}$. The equation of $Y_{Z^{\prime}}$ can be approximated with

$$
\frac{d Y_{Z^{\prime}}}{d x} \approx-\frac{\lambda_{X}}{x^{5}}\left\langle\sigma_{3} v^{2}\right\rangle(2 \pi)^{2} m_{X}^{3}\left(\frac{g_{*}}{90}\right) Y_{Z^{\prime}}^{3} .
$$

An approximate solution of eq. (3.16) in $x>x_{c}$ is given by

$$
Y_{Z^{\prime}}(x) \approx \frac{\bar{Y}_{Z^{\prime}}\left(x_{c}\right)\left(1+\delta_{c}\right)}{\sqrt{1+\frac{x_{c}\left(1+\delta_{c}\right)}{2 \delta_{c}}\left(r_{X Z^{\prime}}^{-1 / 2}-\frac{3}{2 x_{a}}\right)\left(1-\frac{x_{c}^{4}}{x^{4}}\right)}} .
$$

We can see that $Y_{Z^{\prime}}(x)$ quickly tends to a fixed quantity after $x>x_{c}$. After $Z^{\prime}$ freezes out, the process $X^{*}+X \leftrightarrow Z^{\prime}+Z^{\prime}$ is still efficient and thus the DM and catalyst are in chemical equilibrium. The distribution of $X$ can be determined by

$$
Y_{X}(x) \approx \frac{\bar{Y}_{X}}{\bar{Y}_{Z^{\prime}}} Y_{Z^{\prime}} \approx 2 r_{X Z^{\prime}}^{3 / 4} e^{-\left(1-r_{X Z^{\prime}}^{-1 / 2}\right) x} Y_{Z^{\prime}}(x)
$$

Since $m_{Z^{\prime}}<m_{X}$, the reaction rate of process $Z^{\prime}+Z^{\prime} \rightarrow X^{*}+X$ is exponentially decreasing and it finally fades out. To be precise, this happens when

$$
\frac{1}{2}\left\langle\sigma_{2} v\right\rangle \bar{n}_{\bar{n}_{Z^{\prime}}^{2}}^{2} n_{Z^{\prime}}^{2} \lesssim 2\left\langle\sigma_{3} v^{2}\right\rangle n_{Z^{\prime}}^{3} \Rightarrow e^{-2\left(1-r_{X Z^{\prime}}^{-1 / 2}\right) x} \lesssim\left[\frac{4\left\langle\sigma_{3} v^{2}\right\rangle}{\left\langle\sigma_{2} v\right\rangle} m_{X}^{3}\left(\frac{2 \pi^{2} g_{*}}{45}\right) Y_{Z^{\prime}}\right] x^{-3}
$$

The exponential function in the left-handed side decreases much faster than $x^{-3}$ in the right-handed side when $x$ grows, and thus $3 Z^{\prime} \rightarrow X^{*}+X$ becomes more efficient than $2 Z^{\prime} \rightarrow X^{*}+X$ in lower temperature, so that we can neglect the $2 Z^{\prime} \rightarrow X^{*}+X$ processes in the catalyzed annihilation era. After that, the equation of $Y_{X}$ becomes

$$
\frac{d Y_{X}}{d x} \approx \frac{\lambda_{X}}{x^{2}}\left[-\frac{1}{2}\left\langle\sigma_{2} v\right\rangle Y_{X}^{2}+2\left\langle\sigma_{3} v^{2}\right\rangle s Y_{Z^{\prime}}^{3}\right]
$$


The DM depletes through $X^{*}+X \rightarrow Z^{\prime}+Z^{\prime}$ and $Z^{\prime}+Z^{\prime}+Z^{\prime} \rightarrow X^{*}+X$ processes which means the catalyzed annihilation stage starts. $Y_{X}$ in this era is given by

$$
\tilde{Y}_{X}(x)=C_{X} x^{-3 / 2},
$$

where

$$
C_{X} \equiv 4 \pi\left(\frac{g_{*}}{90}\right)^{1 / 2}\left(\frac{\left\langle\sigma_{3} v^{2}\right\rangle}{\left\langle\sigma_{2} v\right\rangle}\right)^{1 / 2} m_{X}^{3 / 2} Y_{Z^{\prime}}^{3 / 2} .
$$

The catalyzed annihilation stage ends when

$$
\left\langle\sigma_{2} v\right\rangle n_{X}^{2} \simeq\left\langle\sigma_{3} v\right\rangle n_{Z^{\prime}}^{3} \simeq H n_{X}
$$

and then DM freezes out. There is a good approximate solution of eq. (3.19):

$$
Y_{X}(x) \approx \tilde{Y}_{X}(x) f_{X}(z), \quad f_{X}(z) \equiv \frac{K_{\frac{4}{5}}(z)}{K_{\frac{1}{5}}(z)}
$$

with $z \equiv \frac{2 A_{X}}{5 x^{5 / 2}}$, where $A_{X}$ is defined by

$$
A_{X} \equiv \frac{1}{2} \lambda_{X}\left\langle\sigma_{2} v\right\rangle C_{X}
$$

$K_{\alpha}(z)$ is the modified Bessel function of the second kind. We can check that in the large $z$ limit $f_{X}(z) \rightarrow 1$, while in the small $z$ limit $f_{X}(z) \rightarrow[\Gamma(4 / 5) / \Gamma(1 / 5)](z / 2)^{-3 / 5} \propto x^{3 / 2}$. Therefore, $Y_{X}(x)$ traces $\tilde{Y}_{X}(x)$ before DM freezes out $(z \gg 1)$ and approaches a constant after freeze out $(z \ll 1)$. We define $Y_{X}^{\text {fo. }}$ to denote the final value of $Y_{X}(x)$ after DM freezes out and its explicit expression is given by

$$
Y_{X}^{f o .}=\frac{\Gamma(4 / 5)}{\Gamma(1 / 5)}\left(\frac{A_{X}}{5}\right)^{-3 / 5} .
$$

Finally, the relic abundance of DM today can be computed by

$$
\Omega_{X} h^{2}=2.83 \times 10^{11} \times\left(\frac{m_{X}}{1 \mathrm{TeV}}\right) Y_{X}^{f o} .
$$

We solve the Boltzmann equations numerically for two different benchmark models:

1. $m_{X}=1 \mathrm{TeV}, r_{X Z^{\prime}}^{1 / 2}=m_{X} / m_{Z^{\prime}}=1.32, g_{D}=1.015, t_{\epsilon}=10^{-11}, \xi_{X}=\xi_{Z^{\prime}}=10^{-5}$ (magenta lines of left panel in figure 3 ),

2. $m_{X}=6 \mathrm{TeV}, r_{X Z^{\prime}}^{1 / 2}=m_{X} / m_{Z^{\prime}}=1.25, g_{D}=2.55, t_{\epsilon}=10^{-11}, \xi_{X}=\xi_{Z^{\prime}}=10^{-5}$ (blue lines of left panel in figure 3 ),

which can reproduce the observed relic abundance of DM $\Omega_{X} h^{2} \approx 0.12$ [51]. The evolution of the $Y_{Z^{\prime}}(x)$ and $Y_{X}(x)$ are shown in the left panel of figure 3 . Solid lines represent $Y_{X}(x)$, while the dashed lines represent $Y_{Z^{\prime}}(x)$. The black dotted lines represent the analytical approximate solutions of $Y_{X}(x)$ given by eq. (3.23). We find that our approximate results match the numerical ones very well. The temperature of $Z^{\prime}$ freezing is around $T_{c} \approx m_{X} / 16$. 

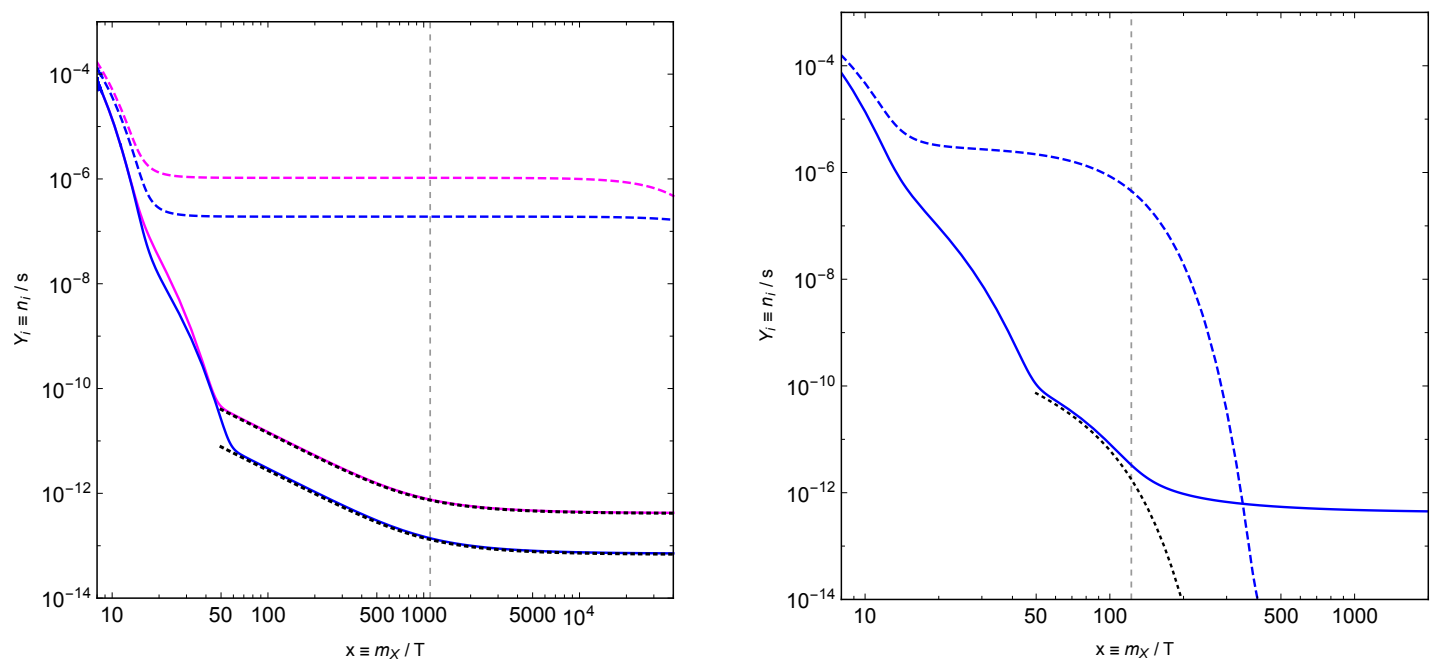

Figure 3. The evolutions of $Y_{Z^{\prime}}(x)$ (dashed lines) and $Y_{X}(x)$ (solid lines). In the left panel, $t_{\epsilon}=10^{-11}, \xi_{X}=\xi_{Z^{\prime}}=10^{-5}$ is chosen for the three benchmark models with $\left(m_{X}, r_{X Z^{\prime}}^{1 / 2}, g_{D}\right)=$ $(1 \mathrm{TeV}, 1.32,1.015)$ (magenta lines), and $(6 \mathrm{TeV}, 1.25,2.55)$ (blue lines). The black dotted lines are the approximated results of $Y_{X}(x)$ given by eq. (3.23). In the right panel, $m_{X}=1 \mathrm{TeV}, r_{X Z^{\prime}}^{1 / 2}=$ 1.3, $g_{D}=0.68, t_{\epsilon}=5 \times 10^{-9}, \xi_{X}=\xi_{Z^{\prime}}=10^{-5}$ is chosen. The black dotted line is the approximate solution of $Y_{X}(x)$ given by eq. (3.32).

The temperature of DM freeze-out is about $T_{f} \approx m_{X} / 10^{3}$ (vertical dashed line in figure 3 ) given by $z \approx 0.3$ ( where $Y_{X}(x)$ is about 1.6 times of $\tilde{Y}_{X}(x)$ ).

Now we can determine the constraint on the decay width of the catalyst. The condition is

$$
\left\langle\Gamma_{Z^{\prime}}\right\rangle \ll H\left(T_{f}\right) \quad \Rightarrow \quad t_{\epsilon} \ll 2 \times 10^{-10},
$$

for the three chosen benchmark models. In the case with $m_{X}=1 \mathrm{TeV}$, current direct detection bound on the magnetic moment of DM is about [40]

$$
\left|\frac{\mu_{X}}{\mu_{N}}\right| \lesssim 10^{-5}
$$

where $\mu_{N}=e / 2 m_{p}$ is the proton magnetic moment. The dark matter magnetic moment can be estimated by $\mu_{X} \sim\left(e / 2 m_{X}\right)\left(g_{D} \hat{c}_{W} s_{\epsilon} / 2\right)$ and thus the bound on the $s_{\epsilon} \approx t_{\epsilon}$ is about

$$
s_{\epsilon} \lesssim 0.05
$$

which is much looser than the constraint from decay width.

Although the model is unlikely to be constrained by the DM direct detection experiment, it can have significant signal in the indirect detection experiments. For example, remnant of DM in dwarfs satellite galaxies can annihilate each other and produce catalysts, and then catalysts will decay into SM particles. These processes can contribute to the continuous spectrum of $\gamma$-ray and then be probed by the Fermi-LAT experiments [9]. The absence of signals put stringent constraints on the parameter space of the models. In figure 4, the dark gray region has been excluded by current Fermi-LAT data, while the 


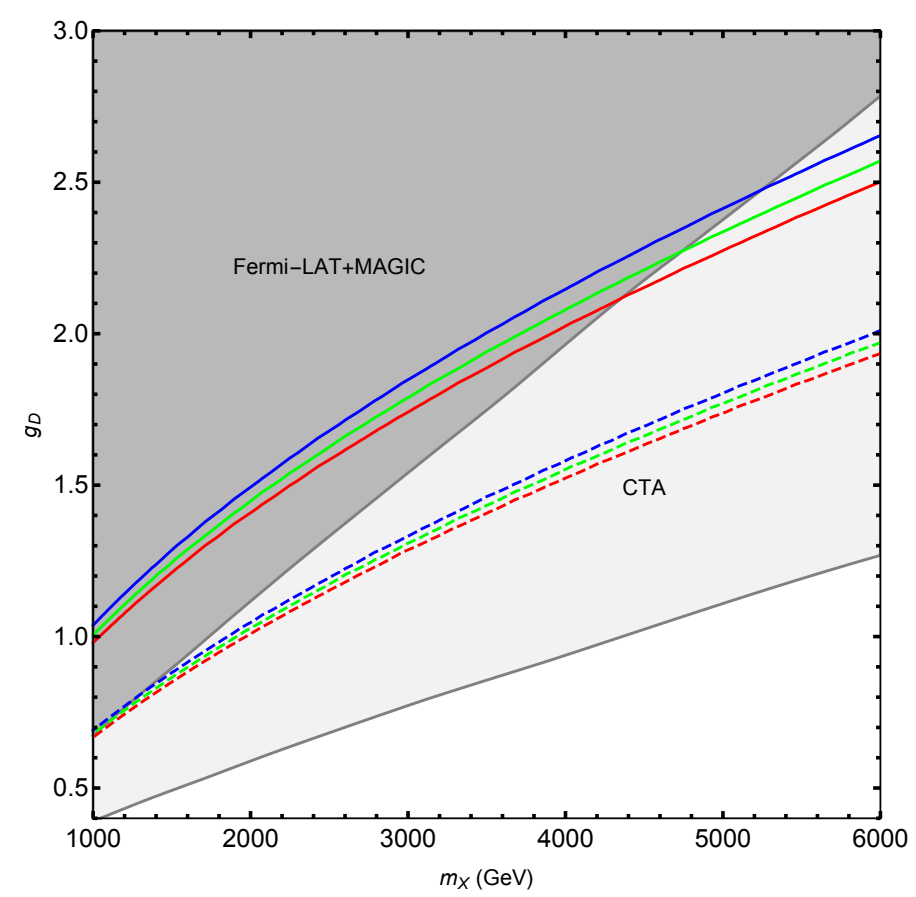

Figure 4. The dark gray region is excluded by Fermi-LAT data [9] at 95\% CL, while the light gray region is the prospect of CTA experiment [45]. Solid lines correspond to the parameters that reproduce $\Omega_{X} h^{2}=0.12$ [51] by choosing $r_{X Z^{\prime}}^{1 / 2}=1.2$ (red), 1.3 (green), and 1.4 (blue) and fixing $t_{\epsilon}=$ $10^{-11}, \xi_{X}=\xi_{Z^{\prime}}=10^{-5}$. Dashed lines represent the cases by fixing $t_{\epsilon}=5 \times 10^{-9}, \xi_{X}=\xi_{Z^{\prime}}=10^{-5}$.

light gray region is an estimation of future CTA experiment sensitivity. The solid colored lines represents the parameters which can obtain the $\Omega_{X} h^{2}=0.12$ for $r_{X Z^{\prime}}^{1 / 2}=1.2$ (red), 1.3 (green), and 1.4 (blue) with fixing $t_{\epsilon}=10^{-11}, \xi_{X}=\xi_{Z^{\prime}}=10^{-5}$. We find that the region $m_{X} \lesssim 4.5 \mathrm{TeV}$ has been excluded by the Fermi-LAT observation at 95\% CL. The whole region of our interest is covered by the prospects of CTA sensitivity [45], so our model can be tested in the next generation of high energy $\gamma$-ray observation.

Note that in the above discussions, we have assumed that the catalyst particle decay after the DM freezes out. When $t_{\epsilon}>10^{-10}$, this assumption is not valid anymore. Consider the case that $Z^{\prime}$ is long-living enough for starting the catalyzed annihilation but it decays before $X$ freezes out. The equation of $Y_{Z^{\prime}}$ becomes

$$
\frac{d Y_{Z^{\prime}}}{d x} \approx-\lambda_{X} \frac{\Gamma_{Z^{\prime}}}{(2 \pi)^{2} m_{X}^{3}}\left(\frac{90}{g_{*}}\right) x\left(Y_{Z^{\prime}}-\bar{Y}_{Z^{\prime}}\right) .
$$

which has an approximate solution of the form

$$
Y_{Z^{\prime}}(x) \approx \tilde{Y}_{Z^{\prime}} e^{-\frac{C_{Z^{\prime}}}{2} x^{2}}
$$

where $\tilde{Y}_{Z^{\prime}}$ is the $x \rightarrow \infty$ limit of $(3.17)$, and $C_{Z^{\prime}} \equiv\left(90 \lambda_{X} \Gamma_{Z^{\prime}}\right) /\left((2 \pi)^{2} g_{*} m_{X}^{3}\right)$. We can see that $Y_{Z^{\prime}}(x)$ starts to fastly decrease when $C_{Z^{\prime}} x^{2} \sim 1$. At the same time, $Y_{X}(x)$ in the catalyzed annihilation era should be

$$
\hat{Y}_{X}(x)=C_{X} x^{-3 / 2} e^{-\frac{3 C_{Z^{\prime}}}{4} x^{2}} .
$$


The freeze-out of $X$ happens when

$$
x_{f} \approx\left(\frac{3}{C_{Z^{\prime}}} W_{0}\left[\left(\frac{2 \delta_{f}\left(2+\delta_{f}\right) A_{X}}{3\left(1+\delta_{f}\right) C_{Z^{\prime}}}\right)^{4 / 9} \frac{C_{Z^{\prime}}}{3}\right]\right)^{1 / 2} .
$$

The approximate result of $Y_{X}(\infty)$ after $x>x_{f}$ is given by

$$
Y_{X}(\infty) \approx \frac{\hat{Y}_{X}\left(x_{f}\right)\left(1+\delta_{f}\right)}{1+\frac{3\left(1+\delta_{f}^{2}\right)}{2\left(2+\delta_{f}\right) \delta_{f}}\left(1+C_{Z^{\prime}} x_{f}^{2}\right)},
$$

where $\delta_{f}=1.3$ can reproduce the numerical result well. In the right panel of figure 3 , we show the evolution of $Y_{X}(x)$ (blue solid) and $Y_{Z^{\prime}}(x)$ (blue dashed) from numerical computation for a benchmark model with $m_{X}=1 \mathrm{TeV}, r_{X Z^{\prime}}^{1 / 2}=1.3, g_{D}=0.68, t_{\epsilon}=$ $5 \times 10^{-9}, \xi_{X}=\xi_{Z^{\prime}}=10^{-5}$. The black dotted line is the approximate solution of $Y_{X}(x)$ before DM freezes out. We can see that the freeze-out of $X$ is triggered by the decay of $Z^{\prime}$, therefore the freeze-out temperature also depends on the decay width of $Z^{\prime}$. In the figure 4 , we show the dashed lines representing the parameters achieving the observed DM relic abundance by choosing $t_{\epsilon}=5 \times 10^{-9}, \xi_{X}=\xi_{Z^{\prime}}=10^{-5}$. The red, green, blue colors corresponds to $r_{X Z^{\prime}}^{1 / 2}=1.2,1.3$, 1.4. Since $g_{D}$ for reproducing the DM relic abundance is smaller in this case, the region with $m_{X}>1 \mathrm{TeV}$ survives from the Fermi-LAT bound. The CTA sensitivity also covers all the dashed lines of the model, so we can expect our models to be tested in the future experiments.

\section{Kinetic equilibrium before DM freeze-out}

As pointed out in ref. [10], it is not easy to keep DM in kinetic equilibrium (KE) with the thermal bath at a temperature as low as $T_{f} \sim m_{X} / 10^{3}$. The reason is that the couplings corresponding to DM annihilation are usually the same as the ones leading to scattering. If the catalyst particle is required to decouple early $\left(T>T_{c} \sim m_{X} / 16\right)$, the corresponding coupling should be very small. On the other hand, a tiny coupling also suppresses the reaction rate of scattering processes, and thus the scattering rate may quickly fall behind the Hubble expansion rate. In previous sections, the annihilation processes of DM to SM particles are mediated by Higgs bosons, whose couplings are set to be as small as $\xi_{X} \sim \xi_{Z^{\prime}} \lesssim 10^{-4}$, and thus the scattering rate of DM with the thermal bath is also extremely suppressed in late time.

In the following discussion, we will discuss two ways for keeping DM in KE until freezeout. One way is to consider a stronger Higgs-portal coupling, and the other way is to couple the DM with a thermal Axion-Like Particle (ALP) $\eta$ during the catalyzed annihilation era.

\subsection{The Higgs-portal strategy}

If the Higgs-portal interaction among the dark and SM sector becomes stronger, it is possible to keep the DM particle in KE until freeze-out. The cross section of scattering 
between $X$ and SM fermion $f$, through the Higgs-portal, is given by

$$
\begin{aligned}
\langle\sigma v\rangle_{X f \rightarrow X f} \approx & \frac{c_{f} g_{D}^{4} v_{2}^{2} m_{f}^{2}}{384 \pi m_{X}^{4} v^{2}}\left[\left(c_{\alpha} \alpha_{13}+s_{\alpha} \alpha_{23}\right)+\frac{4 v_{3}}{v_{2}}\left(c_{\alpha} \alpha_{23}-s_{\alpha} \alpha_{13}\right)\right]^{2} x^{3} \mathcal{F}(x), \\
\mathcal{F}(x)= & \int_{0}^{\infty} d q e^{-x q} \mathcal{I}(x) \\
\mathcal{I}(x) \equiv & \frac{q^{2}}{4(1+2 q)^{2}\left(4 q^{2}+2 r_{h X} q+r_{h X}\right)} \\
& \times\left[8 q^{4}+\left(32-r_{h X}\right) q^{3}-\left(32-26 r_{h X}+12 r_{h X}^{2}\right) q^{2}\right. \\
& \left.-\left(48-32 r_{h X}+12 r_{h X}^{2}\right) q-\left(12-8 r_{h X}+3 r_{h X}^{2}\right)\right] \\
& +\frac{12-8 r_{h X}+3 r_{h X}^{2}}{16} \ln \left(1+\frac{4 q^{2}}{r_{h X}(1+2 q)}\right)
\end{aligned}
$$

where $c_{f}$ is the color number of SM fermion, $f$, while $r_{h X} \equiv m_{1}^{2} / m_{X}^{2}$. The KE condition for DM particle can be estimated by $[52,53]$

$$
\left(\frac{T}{m_{X}}\right) \frac{\Gamma_{e l}}{H} \gtrsim 1, \quad \Gamma_{e l} \approx \sum_{f=\tau, c, b} n_{f}\langle\sigma v\rangle_{X f \rightarrow X f} .
$$

where $H$ is the Hubble expansion rate. Note that a suppression factor $\left(T / m_{X}\right)$ is introduced since a non-relativistic particle requires multiple scatterings with the plasma for transferring its momentum.

Since there are lots of parameters in the potential, we only focus on two special cases. The first case is to consider that $\lambda_{02}=0, s_{\alpha} \ll 1$, and thus the annihilation cross sections of $Z^{\prime}+Z^{\prime} \rightarrow \phi_{\mathrm{SM}}+\phi_{\mathrm{SM}}$ are suppressed by $s_{\alpha}^{2}$ (see eq. (2.17), (2.18), and (3.2)). On the other hand, the scatterings between the DM particle and SM fermions are not suppressed by tiny $s_{\alpha}$ since $c_{\alpha} \alpha_{23}-s_{\alpha} \alpha_{13} \approx-\lambda_{03} v_{3} v / m_{2}^{2}$. Therefore, we can always find a $\lambda_{03}$ which is large enough for maintaining the KE of DM during the catalyzed annihilation era, without affecting the freeze-out of $Z^{\prime}$ in the early era $\left(x_{c} \sim 16\right)$.

For another special choice of parameter that, $\lambda_{03}=0, s_{\alpha} \ll 1$, there is a common Higgs-portal coupling for $X$ and $Z^{\prime}$ since $c_{\alpha} \alpha_{23}-s_{\alpha} \alpha_{13} \approx s_{\alpha}$ is negligible, so that we can simplify the analysis by defining an effective coupling

$$
\xi_{\mathrm{eff}}=\left(\frac{v_{2}}{v}\right)\left(c_{\alpha} \alpha_{13}+s_{\alpha} \alpha_{23}\right) .
$$

The annihilation cross sections for $Z^{\prime}+Z^{\prime} \rightarrow \phi_{\mathrm{SM}}+\phi_{\mathrm{SM}}$ and $X+f \rightarrow X+f$ become

$$
\begin{aligned}
\langle\sigma v\rangle_{Z^{\prime} Z^{\prime} \rightarrow 2 \phi_{\mathrm{SM}}} & \approx \frac{g_{D}^{4} \xi_{\mathrm{eff}}^{2}}{256 \pi m_{Z^{\prime}}^{2}}\left(\frac{m_{t}^{2}}{m_{Z^{\prime}}^{2}}+\frac{4}{3}\right), \\
\langle\sigma v\rangle_{X f \rightarrow X f} & \approx \frac{g_{D}^{4} \xi_{\mathrm{eff}}^{2} c_{f} m_{f}^{2}}{384 \pi m_{X}^{4}} \cdot x^{3} \mathcal{F}(x) .
\end{aligned}
$$

Since a larger $\xi_{\text {eff }}$ can give rise to a more efficient annihilation rate for the catalyst particles, it is possible that the freeze-out temperature of $Z^{\prime}$ is also determined by 2 to 2 annihilation, $Z^{\prime}+Z^{\prime} \rightarrow \phi_{\mathrm{SM}}+\phi_{\mathrm{SM}}$, rather than only by $Z^{\prime}+Z^{\prime}+Z^{\prime} \rightarrow X^{*}+X$. These additional 

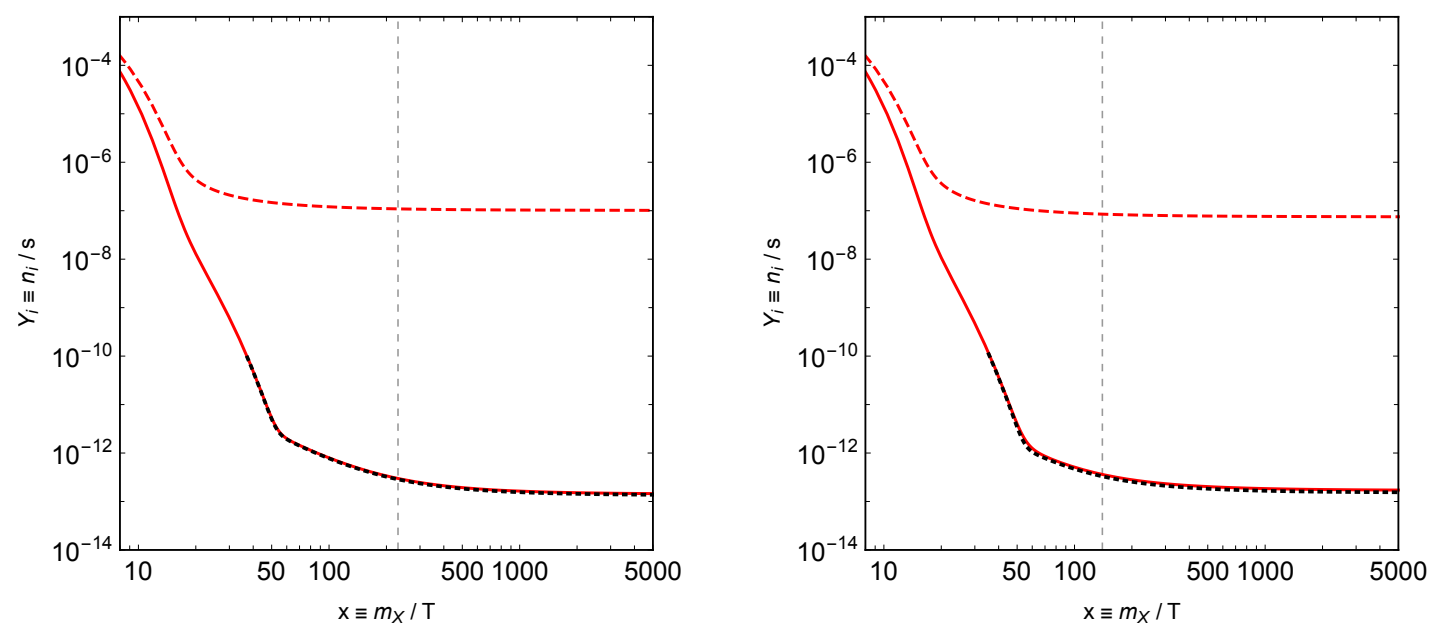

Figure 5. The evolutions of $Y_{Z^{\prime}}(x)$ (red dashed line) and $Y_{X}(x)$ (red solid line). The black dotted line is given by the approximate solution. The vertical gray dashed lines indicate the $x_{f}$ for DM particle. Left panel: Higgs-portal model with parameters, $m_{X}=3 \mathrm{TeV}, r_{X Z^{\prime}}^{1 / 2}=$ $1.3, g_{D}=1.24, t_{\epsilon}=10^{-11}, \xi_{\text {eff }}=1.5 \times 10^{-3}$. Right panel: ALP-portal model with parameters, $m_{X}=2.5 \mathrm{TeV}, r_{X Z^{\prime}}^{1 / 2}=1.3, g_{D}=1.0, m_{X} / \Lambda^{\prime}=0.036, t_{\epsilon}=10^{-11}, \xi_{X}=\xi_{Z^{\prime}}=10^{-5}$.

annihilation channels also accelerate the freeze-out of DM since $\tilde{Y}_{Z^{\prime}}$ is reduced. In the left panel of figure 5, we show the evolution of $Y_{X, Z^{\prime}}$ for a benchmark model with parameters: $m_{X}=3 \mathrm{TeV}, r_{X Z^{\prime}}^{1 / 2}=1.3, g_{D}=1.24, t_{\epsilon}=10^{-11}, \xi_{\text {eff }}=1.5 \times 10^{-3}$. The freeze-out temperature of $\mathrm{DM}$ is found to be $x_{f} \approx 230 \Rightarrow T_{f} \approx 13 \mathrm{GeV}$.

In the left panel of figure 6 , the indirect detection, KE bound, ${ }^{7}$ and five contours reproducing $\Omega_{X} h^{2}=0.12$ are shown in the $m_{X}-g_{D}$ plane. Five colored lines represent models with $\xi_{\text {eff }}=10^{-4}$ (red), $5 \times 10^{-4}$ (magenta), $10^{-3}$ (green), $2 \times 10^{-3}$ (blue), $4 \times 10^{-3}$ (purple), by fixing $r_{X Z^{\prime}}^{1 / 2}=1.3, t_{\epsilon}=10^{-11}$. We find that for $\xi_{\text {eff }} \gtrsim 10^{-3}$, Higgs-portal scattering allows DM to freeze out before out of $\mathrm{KE}$ in our interested parameter region. For $\xi_{\text {eff }}>2 \times 10^{-3}$, DM can be as light as $m_{X}=1.4 \mathrm{TeV}$.

Note that for the case with $\xi_{\text {eff }} \lesssim 10^{-3}$, it is also possible to maintain the KE of $X$ before freeze-out if the catalyst $Z^{\prime}$ decays earlier (but it should be guaranteed to not decay before the catalyzed annihilation starts). This can be understood since the decay of $Z^{\prime}$ leads to a sudden freeze-out of $X$, so that $X$ can easily freezes out before kinetic decoupling.

\subsection{The ALP strategy}

Another way to postpone the kinetic decoupling of DM is considering an axion-like particle (ALP) extension. The ALP couples to the SM and the dark sector in the following form

$$
\mathcal{L}_{\eta} \supset-\frac{\eta}{\Lambda^{\prime}} \tilde{V}_{\mu \nu}^{a} V^{a, \mu \nu}-C_{f} \sum_{f=q, l, \nu} \frac{1}{\Lambda^{\prime}}\left(\partial_{\mu} \eta\right) \bar{f} \gamma^{\mu} \gamma^{5} f+\ldots
$$

\footnotetext{
${ }^{7}$ Since the KE bound also depends on $\xi_{\text {eff }}$, its limit line (dashed brown) is obtained by connecting the bound points for different $\xi_{\text {eff }}$.
} 
where we have simplified our model by assuming that $C_{f}$ is common for all species of SM fermions, and the coupling between $\eta$ and SM gauge bosons are negligible. If the ALP has a mass around $1 \mathrm{GeV},{ }^{8}$ it can easily keep in thermal equilibrium due to its decay and inverse decay. The cross sections of scattering processes $X+\eta \rightarrow X+\eta$ and $X+f \rightarrow X+f$ can be derived as

$$
\begin{aligned}
\langle\sigma v\rangle_{X \eta} & \approx \frac{4 m_{X}^{2}}{27 \pi \Lambda^{\prime 4}} x^{-2} \\
\langle\sigma v\rangle_{X f} & \approx \frac{8 c_{f}\left(C_{f}\right)^{2} m_{f}^{2}}{3 \pi \Lambda^{\prime 2}}=\langle\sigma v\rangle_{X \eta} \times 18 c_{f}\left(C_{f}\right)^{2}\left(\frac{m_{f}}{T}\right)^{2}
\end{aligned}
$$

where $c_{f}$ is the color number of SM fermion, $f$. Taking all dominant fermion species, $\tau, c$ and $b$ into account, the collision rate of $X+f \rightarrow X+f$ scattering is

$$
\Gamma_{X f}=\sum_{f=\tau, c, b} n_{f}\langle\sigma v\rangle_{X f} \approx \Gamma_{X \eta} C_{f}^{2}\left(\frac{47 \mathrm{GeV}}{T}\right)^{2}, \quad\left(T \gtrsim m_{b}\right)
$$

where $\Gamma_{X \eta}=n_{\eta}\langle\sigma v\rangle_{X \eta}$ is the collision rate of $X-\eta$ scattering. If we take $C_{f}=1$, we can see that the $X+\eta \rightarrow X+\eta$ scattering dominates in a temperature $T \gtrsim 50 \mathrm{GeV}$, while the $X+f \rightarrow X+f$ scatterings dominate when the temperature drops below $50 \mathrm{GeV}$. The requirement that kinetic equilibrium is maintained until DM freeze-out leads to a condition:

$$
\left(\frac{T}{m_{X}}\right) \frac{\Gamma_{X \eta}+\Gamma_{X f}}{H} \gtrsim 1
$$

at $T=T_{f}=m_{X} / x_{f}$.

On the other hand, the annihilation rates of $Z^{\prime}+Z^{\prime} \rightarrow \eta+\eta$ and $Z^{\prime}+Z^{\prime} \rightarrow \eta \rightarrow \bar{t}+t$ at $x_{c}$ are given by

$$
\begin{aligned}
\langle\sigma v\rangle_{2 Z^{\prime} \rightarrow 2 \eta} \bar{n}_{Z^{\prime}} & \approx \frac{r_{X Z^{\prime}}^{-1}}{18 \pi m_{X}^{2}}\left(\frac{m_{X}}{\Lambda^{\prime}}\right)^{4} \times 3 \times \frac{m_{X}^{3}}{(2 \pi)^{3 / 2}} x_{c}^{-3 / 2} e^{-x_{c}}, \\
\langle\sigma v\rangle_{2 Z^{\prime} \rightarrow \bar{t} t} \bar{n}_{Z^{\prime}} & \approx \frac{4 C_{f}^{2} m_{t}^{2}}{\pi \Lambda^{\prime 4}} x_{c}^{-1} \times 3 \times \frac{m_{X}^{3}}{(2 \pi)^{3 / 2}} x_{c}^{-3 / 2} e^{-x_{c}},
\end{aligned}
$$

which are usually larger than the rate of $Z^{\prime}+Z^{\prime}+Z^{\prime} \rightarrow X^{*}+X$. It means that the freeze-out temperature of $Z^{\prime}$ is determined by the effective ALP scale, $\Lambda^{\prime}$, rather than by the gauge couping, $g_{D}$. Since $Y_{Z^{\prime}}$ directly affect the evolution of $Y_{X}$ during the catalyzed annihilation era, the final relic density of DM also depends on $m_{X} / \Lambda^{\prime}$. In the right panel of figure 5, we show the evolutions of $Y_{X}$ and $Y_{Z^{\prime}}$ for a benchmark model with parameters: $m_{X}=2.5 \mathrm{TeV}, r_{X Z^{\prime}}^{1 / 2}=1.3, g_{D}=1.0, m_{X} / \Lambda^{\prime}=0.036, t_{\epsilon}=10^{-11}, \xi_{X}=\xi_{Z^{\prime}}=10^{-5}$. We find that $Z^{\prime}$ freeze-out at $x_{c} \approx 20$ and the catalyzed annihilation of DM occurs in $50 \lesssim x \lesssim x_{f} \approx 140$. In the right panel of figure 6 , we show the constraints of indirect detection and KE in the $m_{X}-g_{D}$ plane. The colored solid lines represent the parameters which can reproduce the relic abundance of DM. The corresponding parameters are chosen to be $m_{X} / \Lambda^{\prime}=0.020$ (red), 0.025 (magenta), 0.030 (green), 0.035 (blue), 0.040 (purple) by fixing $r_{X Z^{\prime}}^{1 / 2}=1.3, t_{\epsilon}=10^{-11}, \xi_{X}=\xi_{Z^{\prime}}=10^{-5}$. We find that for the mass region, $m_{X} \geq 1 \mathrm{TeV}, m_{X} / \Lambda^{\prime} \gtrsim 0.030$ is required for maintaining the $\mathrm{KE}$ of DM before freeze-out.

\footnotetext{
${ }^{8} \mathrm{An}$ ALP with a mass $\sim 1 \mathrm{GeV}$ and $\Lambda^{\prime} \gtrsim 30 \mathrm{TeV}$ is consistent with most of current experimental constraints [54].
} 

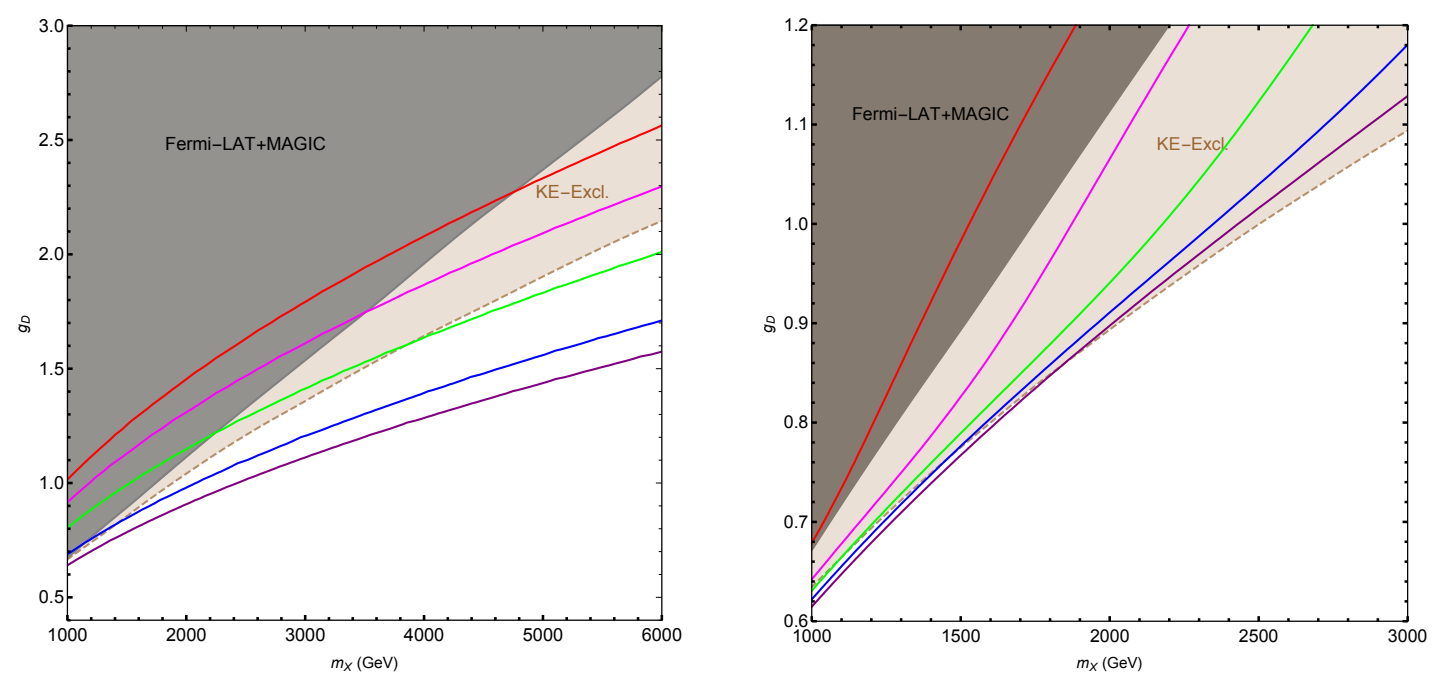

Figure 6. The dark gray region is excluded by Fermi-LAT data [9] at 95\% CL, while the brown region is excluded by out of kinetic equilibrium of DM. Solid lines correspond to the parameters which can reproduce $\Omega_{X} h^{2}=0.12$ [51]. Left panel: colored lines indicate $\xi_{\text {eff }}=10^{-4}$ (red), $5 \times 10^{-4}$ (magenta), $10^{-3}$ (green), $2 \times 10^{-3}$ (blue), $4 \times 10^{-3}$ (purple) by fixing $r_{X Z^{\prime}}^{1 / 2}=1.3, t_{\epsilon}=10^{-11}$. Right panel: colored lines indicate $m_{X} / \Lambda^{\prime}=0.020$ (red), 0.025 (magenta), 0.030 (green), 0.035 (blue), 0.040 (purple) by fixing $r_{X Z^{\prime}}^{1 / 2}=1.3, t_{\epsilon}=10^{-11}, \xi_{X}=\xi_{Z^{\prime}}=10^{-5}$.

\section{Conclusion}

In this work, we propose a vector dark matter (DM) model in which the DM relic density is determined by the catalyzed freeze-out mechanism. In our model, the DM candidate $X_{\mu}$ and a catalyst $Z_{\mu}^{\prime} \approx C_{\mu}$ are unified into the dark $\mathrm{SU}(2)_{D}$ gauge fields. The $\mathrm{SU}(2)_{D}$ gauge symmetry is spontaneously broken by VEVs of a doublet and a real triplet scalar fields. Since the catalyst only acquires its mass from the doublet while the DM acquires its mass from both the doublet and triplet, the catalyst is automatically lighter than the DM. The mass condition $3 m_{Z^{\prime}}>2 m_{x}$ for the process $Z^{\prime}+Z^{\prime}+Z^{\prime} \rightarrow X^{*}+X$ can also be naturally achieved if the VEVs of the scalar fields satisfy $v_{3} \lesssim 0.56 v_{2}$. Since the catalyzed freeze-out mechanism requires the catalyst to decay after the DM freezes out, we need to introduce a dimension-5 operator $B^{\mu \nu} \Delta^{a} V_{\mu \nu}^{a}$ to break a discrete symmetry $G_{D}$. Such an operator can be easily induced in one loop level by introducing a super heavy fermionic doublet of $\mathrm{SU}(2)_{D}$.

We derive the annihilation cross sections of all the relevant processes, especially, $X^{*}+X \rightarrow Z^{\prime}+Z^{\prime}$ and $Z^{\prime}+Z^{\prime}+Z^{\prime} \rightarrow X^{*}+X$. Then we develop an analytical approximate solution to the Boltzmann equations and compare them to numerical computations. We find that our approximate solution works well so we use them to discuss the constraints from cosmological and astrophysical observations. We provide three benchmark models in which the observed dark matter relic abundance can be achieved. The direct detection constraint is weak in our models since small Higgs-portal couplings can be chosen. However, this model predicts relatively strong DM annihilation cross section, and thus indirect detection experiments can put stringent constraints on it. We find that the $\gamma$-ray spectrum from the 
Fermi-LAT experiment has excluded the mass region of $m_{X}<1.2 \mathrm{TeV}$ for the models with a long-living catalyst. On the other hand, In a model that the catalyst decay during the catalyzed annihilation era, the Fermi-LAT constraint gets looser since a smaller gauge coupling $g_{D}$ is required by the DM relic abundance. We also find that our model can be tested in the next generation of high energy $\gamma$-ray observations, such as the CTA experiment.

All these discussions are based on an assumption that the DM are kept in kinetic equilibrium (KE) with the thermal bath. However, no concrete mechanism is known to be capable of achieving the $\mathrm{KE}$ in such a late time (about $T \sim m_{X} / 10^{3}$ ). In the last section, we propose two paradigms which can partially solve the problem. In the first paradigm, DM particle maintains $\mathrm{KE}$ with the plasma via $X$-fermions scattering mediated by the Higgs boson. In the second paradigm, we introduce a thermal axion-like particle (ALP), which can collide with DM particles directly, and also plays the role of a mediator of $X$-fermions scattering. In both paradigms, there are parameter spaces allowing DM particles to keep in KE with the plasma until the DM freeze-out. The price we need to pay is that the freeze-out temperature of the catalyst is determined by an extra parameter in both cases. The DM freeze-out via catalyzed annihilation still works, but it implies a freeze-out temperature with $x_{f} \sim \mathcal{O}(100)$, which is about an order of magnitude higher than that from the original model.

\section{Acknowledgments}

This work is supported by the National Natural Science Foundation of China (NSFC) under Grant Nos. 11875327 and 11905300, the Fundamental Research Funds for the Central Universities, the Natural Science Foundation of Guangdong Province, and the Sun Yat-Sen University Science Foundation.

Open Access. This article is distributed under the terms of the Creative Commons Attribution License (CC-BY 4.0), which permits any use, distribution and reproduction in any medium, provided the original author(s) and source are credited.

\section{References}

[1] LUX collaboration, Results from a search for dark matter in the complete LUX exposure, Phys. Rev. Lett. 118 (2017) 021303 [arXiv: 1608.07648] [INSPIRE].

[2] XENON collaboration, Dark matter search results from a one ton-year exposure of XENON1T, Phys. Rev. Lett. 121 (2018) 111302 [arXiv:1805.12562] [INSPIRE].

[3] PandaX-4T collaboration, Dark matter search results from the PandaX-4T commissioning run, Phys. Rev. Lett. 127 (2021) 261802 [arXiv:2107.13438] [INSPIRE].

[4] L.E. Strigari, Galactic searches for dark matter, Phys. Rept. 531 (2013) 1 [arXiv: 1211.7090] [INSPIRE].

[5] PAMELA collaboration, Cosmic-ray positron energy spectrum measured by PAMELA, Phys. Rev. Lett. 111 (2013) 081102 [arXiv: 1308.0133] [INSPIRE].

[6] Fermi-LAT collaboration, Searching for dark matter annihilation from Milky Way dwarf spheroidal galaxies with six years of Fermi Large Area Telescope data, Phys. Rev. Lett. 115 (2015) 231301 [arXiv: 1503.02641] [INSPIRE]. 
[7] FERMI-LAT and DES collaborations, Searching for dark matter annihilation in recently discovered Milky Way satellites with Fermi-LAT, Astrophys. J. 834 (2017) 110 [arXiv: 1611.03184] [INSPIRE].

[8] S. Profumo, F.S. Queiroz, J. Silk and C. Siqueira, Searching for secluded dark matter with H.E.S.S., Fermi-LAT and Planck, JCAP 03 (2018) 010 [arXiv:1711.03133] [INSPIRE].

[9] S. Hoof, A. Geringer-Sameth and R. Trotta, A global analysis of dark matter signals from 27 dwarf spheroidal galaxies using 11 years of Fermi-LAT observations, JCAP 02 (2020) 012 [arXiv: 1812.06986] [INSPIRE].

[10] C.-Y. Xing and S.-H. Zhu, Dark matter freeze-out via catalyzed annihilation, Phys. Rev. Lett. 127 (2021) 061101 [arXiv:2102.02447] [INSPIRE].

[11] M. Pospelov, A. Ritz and M.B. Voloshin, Secluded WIMP dark matter, Phys. Lett. B 662 (2008) 53 [arXiv:0711.4866] [INSPIRE].

[12] N. Arkani-Hamed, D.P. Finkbeiner, T.R. Slatyer and N. Weiner, A theory of dark matter, Phys. Rev. D 79 (2009) 015014 [arXiv:0810.0713] [InSPIRE].

[13] Y. Hochberg, E. Kuflik, H. Murayama, T. Volansky and J.G. Wacker, Model for thermal relic dark matter of strongly interacting massive particles, Phys. Rev. Lett. 115 (2015) 021301 [arXiv:1411.3727] [INSPIRE].

[14] Y. Hochberg, E. Kuflik, T. Volansky and J.G. Wacker, Mechanism for thermal relic dark matter of strongly interacting massive particles, Phys. Rev. Lett. 113 (2014) 171301 [arXiv: 1402.5143] [INSPIRE].

[15] N. Bernal and X. Chu, Z 2 SIMP dark matter, JCAP 01 (2016) 006 [arXiv:1510.08527] [INSPIRE].

[16] N. Bernal, X. Chu and J. Pradler, Simply split strongly interacting massive particles, Phys. Rev. D 95 (2017) 115023 [arXiv:1702.04906] [INSPIRE].

[17] J. Smirnov and J.F. Beacom, New freezeout mechanism for strongly interacting dark matter, Phys. Rev. Lett. 125 (2020) 131301 [arXiv:2002.04038] [INSPIRE].

[18] Y. Farzan and A.R. Akbarieh, VDM: a model for Vector Dark Matter, JCAP 10 (2012) 026 [arXiv:1207.4272] [INSPIRE].

[19] T. Abe, M. Kakizaki, S. Matsumoto and O. Seto, Simple vector WIMP dark matter, PoS DSU2012 (2012) 050 [INSPIRE].

[20] G. Arcadi, A. Djouadi and M. Kado, The Higgs-portal for vector dark matter and the effective field theory approach: a reappraisal, Phys. Lett. B 805 (2020) 135427 [arXiv:2001.10750].

[21] S. Baek, P. Ko, W.-I. Park and E. Senaha, Higgs portal vector dark matter: revisited, JHEP 05 (2013) 036 [arXiv: 1212.2131] [InSPIRE].

[22] C.-R. Chen, Y.-K. Chu and H.-C. Tsai, An elusive vector dark matter, Phys. Lett. B 741 (2015) 205 [arXiv:1410.0918] [InSPIRE].

[23] A. DiFranzo, P.J. Fox and T.M.P. Tait, Vector dark matter through a radiative Higgs portal, JHEP 04 (2016) 135 [arXiv:1512.06853] [INSPIRE].

[24] O. Lebedev, H.M. Lee and Y. Mambrini, Vector Higgs-portal dark matter and the invisible Higgs, Phys. Lett. B 707 (2012) 570 [arXiv:1111.4482] [INSPIRE].

[25] M. Duch, B. Grzadkowski and M. McGarrie, A stable Higgs portal with vector dark matter, JHEP 09 (2015) 162 [arXiv: 1506.08805] [INSPIRE]. 
[26] D. Hadjimichef, Massive dark photons in a Higgs portal model, AIP Conf. Proc. 1693 (2015) 050012 [arXiv: 1601.00293] [INSPIRE].

[27] M. Zaazoua, L. Truong, K.A. Assamagan and F. Fassi, Higgs portal vector dark matter interpretation: review of effective field theory approach and ultraviolet complete models, arXiv:2107.01252 [INSPIRE].

[28] P. Adshead and K.D. Lozanov, Self-gravitating vector dark matter, Phys. Rev. D 103 (2021) 103501 [arXiv:2101.07265] [INSPIRE].

[29] T. Hambye, Hidden vector dark matter, JHEP 01 (2009) 028 [arXiv:0811.0172] [INSPIRE].

[30] T. Hambye and M.H.G. Tytgat, Confined hidden vector dark matter, Phys. Lett. B 683 (2010) 39 [arXiv: 0907.1007] [InSPIRE].

[31] S. Baek, P. Ko and W.-I. Park, Hidden sector monopole, vector dark matter and dark radiation with Higgs portal, JCAP 10 (2014) 067 [arXiv:1311.1035] [INSPIRE].

[32] H. Davoudiasl and I.M. Lewis, Dark matter from hidden forces, Phys. Rev. D 89 (2014) 055026 [arXiv: 1309.6640] [INSPIRE].

[33] C. Gross, O. Lebedev and Y. Mambrini, Non-Abelian gauge fields as dark matter, JHEP 08 (2015) 158 [arXiv: 1505.07480] [INSPIRE].

[34] C.-H. Chen and T. Nomura, $\mathrm{SU}(2)_{X}$ vector DM and galactic center gamma-ray excess, Phys. Lett. B 746 (2015) 351 [arXiv: 1501.07413] [INSPIRE].

[35] A. Karam and K. Tamvakis, Dark matter and neutrino masses from a scale-invariant multi-Higgs portal, Phys. Rev. D 92 (2015) 075010 [arXiv:1508.03031] [INSPIRE].

[36] A. Karam and K. Tamvakis, Dark matter from a classically scale-invariant $\mathrm{SU}(3)_{X}$, Phys. Rev. D 94 (2016) 055004 [arXiv: 1607.01001] [INSPIRE].

[37] P. Ko and Y. Tang, Residual non-Abelian dark matter and dark radiation, Phys. Lett. B $\mathbf{7 6 8}$ (2017) 12 [arXiv: 1609.02307] [INSPIRE].

[38] S.-M. Choi et al., Vector SIMP dark matter, JHEP 10 (2017) 162 [arXiv:1707.01434] [INSPIRE].

[39] T. Abe, M. Fujiwara, J. Hisano and K. Matsushita, A model of electroweakly interacting non-Abelian vector dark matter, JHEP 07 (2020) 136 [arXiv:2004.00884] [INSPIRE].

[40] J. Hisano, A. Ibarra and R. Nagai, Direct detection of vector dark matter through electromagnetic multipoles, JCAP 10 (2020) 015 [arXiv: 2007.03216] [INSPIRE].

[41] Z. Hu, C. Cai, Y.-L. Tang, Z.-H. Yu and H.-H. Zhang, Vector dark matter from split SU(2) gauge bosons, JHEP 07 (2021) 089 [arXiv: 2103.00220] [INSPIRE].

[42] N. Baouche, A. Ahriche, G. Faisel and S. Nasri, Phenomenology of the hidden SU(2) vector dark matter model, Phys. Rev. D 104 (2021) 075022 [arXiv:2105.14387] [INSPIRE].

[43] A. Belyaev, G. Cacciapaglia, J. McKay, D. Marin and A.R. Zerwekh, Minimal spin-one isotriplet dark matter, Phys. Rev. D 99 (2019) 115003 [arXiv:1808.10464] [INSPIRE].

[44] B. Holdom, Two U(1)'s and $\epsilon$ charge shifts, Phys. Lett. B 166 (1986) 196 [INSPIRE].

[45] CTA Consortium collaboration, Dark matter and fundamental physics with the Cherenkov Telescope Array, Astropart. Phys. 43 (2013) 189 [arXiv:1208.5356] [INSPIRE].

[46] K.S. Babu, C.F. Kolda and J. March-Russell, Implications of generalized Z-Z' mixing, Phys. Rev. D 57 (1998) 6788 [hep-ph/9710441] [INSPIRE]. 
[47] M.T. Frandsen, F. Kahlhoefer, S. Sarkar and K. Schmidt-Hoberg, Direct detection of dark matter in models with a light $Z^{\prime}$, JHEP 09 (2011) 128 [arXiv:1107.2118] [INSPIRE].

[48] J. Lao, C. Cai, Z.-H. Yu, Y.-P. Zeng and H.-H. Zhang, Fermionic and scalar dark matter with hidden U(1) gauge interaction and kinetic mixing, Phys. Rev. D 101 (2020) 095031 [arXiv: 2003.02516] [INSPIRE].

[49] J.M. Cline, H. Liu, T. Slatyer and W. Xue, Enabling forbidden dark matter, Phys. Rev. D 96 (2017) 083521 [arXiv: 1702.07716] [inSPIRE].

[50] E. Gabrielli, L. Marzola, M. Raidal and H. Veermäe, Dark matter and spin-1 milli-charged particles, JHEP 08 (2015) 150 [arXiv:1507.00571] [INSPIRE].

[51] Planck collaboration, Planck 2018 results. VI. Cosmological parameters, Astron. Astrophys. 641 (2020) A6 [Erratum ibid. 652 (2021) C4] [arXiv: 1807.06209] [INSPIRE].

[52] S. Hofmann, D.J. Schwarz and H. Stoecker, Damping scales of neutralino cold dark matter, Phys. Rev. D 64 (2001) 083507 [astro-ph/0104173] [INSPIRE].

[53] L. Visinelli and P. Gondolo, Kinetic decoupling of WIMPs: analytic expressions, Phys. Rev. D 91 (2015) 083526 [arXiv: 1501.02233] [INSPIRE].

[54] M. Bauer, M. Neubert and A. Thamm, Collider probes of axion-like particles, JHEP 12 (2017) 044 [arXiv: 1708.00443] [INSPIRE]. 